\title{
BubR1 allelic effects drive phenotypic heterogeneity in mosaic-variegated aneuploidy progeria syndrome
}

\author{
Cynthia J. Sieben, ${ }^{1}$ Karthik B. Jeganathan, ${ }^{2}$ Grace G. Nelson, ${ }^{2}$ Ines Sturmlechner, ${ }^{2}$ Cheng Zhang, ${ }^{3}$ Willemijn H. van Deursen, ${ }^{2}$ \\ Bjorn Bakker, ${ }^{4}$ Floris Foijer, ${ }^{4} \mathrm{Hu} \mathrm{Li},{ }^{3}$ Darren J. Baker, ${ }^{1,2}$ and Jan M. van Deursen ${ }^{1,2}$ \\ 'Departments of Biochemistry and Molecular Biology, ${ }^{2}$ Pediatric and Adolescent Medicine, and ${ }^{3}$ Molecular Pharmacology and Experimental Therapeutics, Mayo Clinic, Rochester, Minnesota, USA. ${ }^{4}$ European \\ Research Institute for the Biology of Aging, University Medical Center Groningen, University of Groningen, Groningen, Netherlands.
}

\begin{abstract}
Mosaic-variegated aneuploidy (MVA) syndrome is a rare childhood disorder characterized by biallelic BUBR1, CEP57, or TRIP13 aberrations; increased chromosome missegregation; and a broad spectrum of clinical features, including various cancers, congenital defects, and progeroid pathologies. To investigate the mechanisms underlying this disorder and its

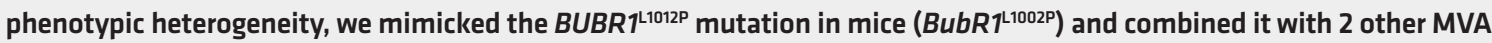
variants, $B U B R 1^{x 753}$ and $B U B R 1^{H}$, generating a truncated protein and low amounts of wild-type protein, respectively. Whereas $B u b R 1^{X 753 / L 1002 P}$ and $B u b R 1^{H / X 753}$ mice died prematurely, BubR $1^{H / L 1002 P}$ mice were viable and exhibited many MVA features, including cancer predisposition and various progeroid phenotypes, such as short lifespan, dwarfism, lipodystrophy, sarcopenia, and low cardiac stress tolerance. Strikingly, although these mice had a reduction in total BUBR1 and spectrum of MVA phenotypes similar to that of $B u b R 1^{H / H}$ mice, several progeroid pathologies were attenuated in severity, which in skeletal muscle coincided with reduced senescence-associated secretory phenotype complexity. Additionally, mice carrying monoallelic BubR1 mutations were prone to select MVA-related pathologies later in life, with predisposition to sarcopenia correlating with mTORC1 hyperactivity. Together, these data demonstrate that BUBR1 allelic effects beyond protein level and aneuploidy contribute to disease heterogeneity in both MVA patients and heterozygous carriers of MVA mutations.
\end{abstract}

\section{Introduction}

Mosaic-variegated aneuploidy (MVA) syndrome is an autosomal recessive syndrome characterized by near-diploid aneuploidies involving multiple tissues and chromosomes (1-4). A wide variety of additional clinical features are associated with this syndrome, including microcephaly, growth and mental retardation, hypothyroidism, facial dysmorphisms, skeletal and renal anomalies, gastrointestinal and cardiac defects, childhood cancers, and early mortality (5). Three genes, all with roles in chromosome segregation, have been implicated in MVA syndrome: the mitotic checkpoint gene BUBR1 (also named $B U B 1 B)(1,2,5)$, the centrosomal protein $C E P 57(3,6)$, and the spindle assembly checkpoint (SAC) activator TRIP13 (4). The majority of patients have bi- or monoallelic mutations in $B U B R 1$, with biallelic alterations typically involving a nonsense mutation in combination with a missense mutation in the kinase domain of the protein $(2,5)$. Patients with monoallelic mutations inherit a nonsense or missense mutation in combination with a hypomorphic allele, without a clear mutation, that yields low amounts of wild-type BUBR1 protein $(1,5)$.

Phenotypic variability is remarkably high among MVA syndrome patients, not only between patients that have mutations

Conflict of interest: The authors have declared that no conflict of interest exists. Copyright: (c) 2020, American Society for Clinical Investigation. Submitted: December 17, 2018; Accepted: September 18, 2019; Published: November 18, 2019

Reference information: J Clin Invest. 2020;130(1):171-188.

https://doi.org/10.1172/JCl126863. in different MVA genes, but also between those with distinct mutations in the same gene (2-5). Although MVA cases with $B U B R 1$ mutations constitute the largest cohort, the overall size of this group is still too small for meaningful genotype-phenotype correlation analyses. One distinguishing feature observed in all MVA patients, regardless of the underlying mutations, is that they inaccurately segregate whole chromosomes, which has prompted speculation that the resulting aneuploidies drive the clinical features of the syndrome (7). However, decisive evidence to support this idea remains elusive. For instance, genetically engineered mice with alterations in chromosomal instability (CIN) genes are often predisposed to tumors, but do not exhibit other clinical phenotypes of MVA syndrome, with the exception of one BubR1based aneuploid model (8).

This particular model was part of a series of mouse mutants with a graded reduction in BUBR1 protein levels created by the use of wild-type (+), hypomorphic (H), and knockout (-) BubR1 alleles. Mutants expressing $0 \%\left(B u b R 1^{-/}\right)$or approximately $5 \%\left(B u b R 1^{-/ H}\right)$ of normal BUBR1 protein levels fail to survive beyond embryonic day 3.5 (E3.5) and postnatal day 1, respectively, but mice expressing approximately $10 \%$ BUBR1 $\left(B u b R 1^{\mathrm{H} / \mathrm{H}}\right)$ are viable (8). Besides near-diploid aneuploidies, these mice exhibit growth retardation, facial dysmorphisms, skeletomuscular, renal, and cardiac anomalies, cataracts, lipodystrophy, and early mortality (8-11). Furthermore, $B u b R 1^{\mathrm{H} / \mathrm{H}}$ mice are sensitive to carcinogen-induced tumors, but do not live long enough to assess predisposition to spontaneous tumors (12). Several of the progeroid phenotypes in $B u b R 1^{\mathrm{H} / \mathrm{H}}$ mice are driven by the accumulation of senescent cells (9-11), which in 
turn led to the identification of cellular senescence as a key contributor to natural aging (13).

Two additional observations have reinforced a possible role for BUBR1 in aspects of natural aging. First, BUBR1 levels decline in various tissues with chronological aging, with geriatric wildtype mice expressing similar amounts of the protein to those of young $B u b R 1^{\mathrm{H} / \mathrm{H}}$ mice (8). Second, transgenic mice that constitutively overexpress BUBR1 are not subject to an age-related decline in BUBR1 and have an increased lifespan with attenuations in muscle and renal atrophy, glomerulosclerosis, cardiac aging, and tumor latency (14). BUBR1 overexpression was also shown to counteract age-related aneuploidization in various tissues, raising the possibility that inaccurate chromosome segregation may be a driver of tissue deterioration with aging. BUBR1 overexpression appears to reinforce both the spindle assembly checkpoint (SAC) and the mitotic error correction machinery, which may underlie the observed reductions in aneuploidization rates (15).

The high clinical heterogeneity among MVA patients with BUBR1 mutations has prompted the idea that BUBR1 is a multitasking protein implicated in a wide variety of biological processes that are differently disrupted, depending on the exact nature of the mutations involved. Early in mitosis, BUBR1, together with MAD2, BUB3, and CDC20, assembles into a potent 4-subunit inhibitor (known as the mitotic checkpoint complex, MCC) of the anaphase-promoting complex (APC/C) that prevents premature anaphase and chromosome missegregation as part of the SAC (16, 17). Once each chromosome has properly and stably attached to the mitotic spindle and sufficient inter-kinetochore tension is generated, the MCC dissociates, allowing APC/C $\mathrm{C}^{\mathrm{CDC} 20}$ to mediate the proteasomal degradation of cyclin $\mathrm{B} 1$ and securin, thereby triggering sister chromatid separation and anaphase onset. BUBR1 also prevents chromosome missegregation as a key component of the Aurora B-driven error correction machinery, which acts to destabilize aberrant microtubule-kinetochore attachments, and through the reactivation of the SAC allows time for proper attachments to occur (18). In this context, BUBR1 localized at mitotic kinetochores acts to recruit PP2A, the phosphatase that counteracts the destabilizing activity of Aurora B kinase (19). Furthermore, BUBR1 regulates clathrin-mediated internalization of the insulin receptor by virtue of its ability to bind to both MAD2 and AP2, thereby quenching signaling through this receptor (20). BUBR1 fulfils this newly discovered function in interphase, further supporting the idea that BUBR1 is a functionally diverse protein with a plethora of mitotic and non-mitotic roles.

Despite significant progress toward understanding these contributions of BUBR1, it remains unclear what the full spectrum of physiologically relevant functions of this protein are, the extent to which the various $B U B R 1$ mutations might perturb these functions, and how all this contributes to the vast clinical heterogeneity within MVA syndrome. To begin to address some of these unresolved questions, we sought to mimic human BUBR1 MVA mutations in mice and characterize the phenotypic consequences. Here, our use of 4 such mutations in various combinations with each other or in combination with a $B u b R 1^{+}$allele demonstrates that subtle allelic effects contribute to disease heterogeneity in both MVA patients and heterozygous carriers of MVA mutations, beyond rates of aneuploidy and BUBR1 protein levels.

\section{Results}

Mice modeling MVA patient BUBR1 $1^{X 753 / L 1012 P}$ die during early embryogenesis. BUBR1 2211insGTTA, a mutant BUBR1 allele that results in a frameshift and yields an unstable truncated protein referred to as BUBR1 ${ }^{\mathrm{X} 753}$, has been identified in 2 biallelic MVA patients, one of which also inherits $B U B R 13035 \mathrm{~T}>\mathrm{C}$, a mutant allele that encodes BUBR1 $1^{\mathrm{L} 1012 \mathrm{P}}$ (2). To model this particular patient, we used a previously established mouse strain in which we mimicked the BUBR1 2211insGTTA allele (21) and a new strain in which we converted the leucine at position 1002 into a proline (human L1012 corresponds to mouse L1002; Figure 1A and Supplemental Figure 1, A-C; supplemental material available online with this article; https://doi.org/10.1172/JCI126863DS1). As expected, heterozygotes carrying the L1002P substitution (referred to as $B u b R 1^{+/ L 1002 P}$ mice) were overtly normal. However, mouse embryonic fibroblasts (MEFs) and tissues from $B u b R 1^{+/ L 1002 P}$ mice contained markedly reduced BUBR1 levels (Figure 1B). Side-by-side comparisons of $B u b R 1^{+/ L 1002 P}, B u b R 1^{+/}$, and $B u b R 1^{+/ X 753} \mathrm{MEFs}$ and tissues implied that the $B u b R 1^{\mathrm{L} 1002 \mathrm{P}}$ allele overall yields very little protein (Figure 1B). However, MEFs and testes from $B u b R 1^{+/-}$and $B u b R 1^{+/ X 753}$ mice had significantly lower BUBR1 protein levels than corresponding tissues from $B u b R 1^{+/ L 1002 P}$ mice, whereas levels were similarly low in the thymus and spleen of all 3 genotypes. The L1002P mutation had no impact on BubR1 transcript stability, but the truncating X753 mutation did, with $B u b R 1^{+/ X 753}$ MEFs yielding transcript levels similar to those of $B u b R 1^{+/-}$MEFs (Supplemental Figure 2A). Assessments of BUBR1 protein stability revealed that proteasomal degradation of BUBR1 ${ }^{\text {L1002P }}$ was elevated (Supplemental Figure 2, $\mathrm{B}$ and $\mathrm{C}$ ), which is in concordance with an earlier study showing that human BUBR1 ${ }^{\mathrm{L} 1012 \mathrm{P}}$ protein is misfolded and therefore less stable (7). Nevertheless, mitotic BubR1 ${ }^{+/ L 1002 P}$ MEFs were still able to accumulate normal amounts of BUBR1 at unattached kinetochores, as demonstrated by immunofluorescence (IF) labeling for BUBR1 (Supplemental Figure 2F). BUBR1 ${ }^{\mathrm{WT}}$ protein stability in $B u b R 1^{+/ X 753}$ MEFs seemed similar to that in $B u b R 1^{+/+}$and $B u b R 1^{+/-}$ MEFs (Supplemental Figure 2, C-E).

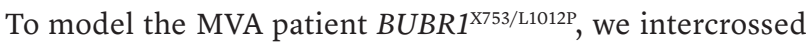
$B u b R 1^{+/ L 1002 P}$ and $B u b R 1^{+/ X 753}$ mice, but no BubR1 $1^{\mathrm{X} 753 / \mathrm{L} 1002 \mathrm{P}}$ mice were identified among 388 newborn pups (Supplemental Table 1). This was unexpected because the patient with this genetic combination was alive for 3.6 months after birth, despite growth retardation and anomalies in a broad spectrum of tissues, including heart, lung, brain, eye, thyroid, and erythrocytes (2). To determine when BubR $1^{\mathrm{X} 753 / \mathrm{L} 1002 \mathrm{P}}$ mice die during embryogenesis, we genotyped a total of 39 E13.5 mice from heterozygous intercrosses, but again no $B u b R 1^{\mathrm{X} 753 / \mathrm{L} 1002 \mathrm{P}}$ mice were observed, whereas $B u b R 1^{+/ L 1002 P}, B u b R 1^{+/ X 753}$, and $B u b R 1^{+/+}$mice were present at expected frequencies (Supplemental Table 1). However, repeating the analysis at E3.5 yielded viable $B u b R 1^{\mathrm{X} 753 / \mathrm{L} 1002 \mathrm{P}}$ embryos, which were overtly indistinguishable from their $B u b R 1^{+/ L 1002 P}, B u b R 1^{+/ X 753}$, and $B u b R 1^{+/+}$counterparts (Supplemental Table 1). Attempts to culture the inner cell mass from $B u b R 1^{\mathrm{X} 753 / \mathrm{L} 1002 \mathrm{P}}$ blastocysts were unsuccessful; however, this was indicative of early embryonic death due to mitotic failure (data not shown). Thus, a mouse model for MVA patient $B U B R 1^{\mathrm{X} 753 / \mathrm{L} 1012 \mathrm{P}}$ is unattainable, most likely due to severe mitotic defects that interfere with early embryogenesis. 
A

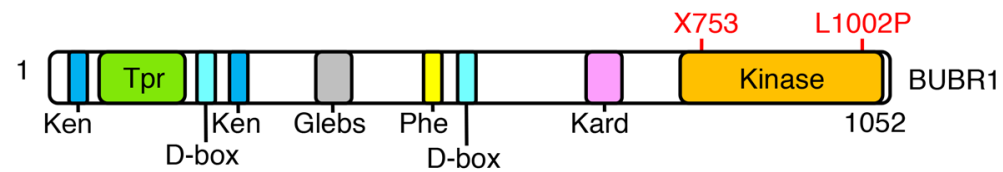

B
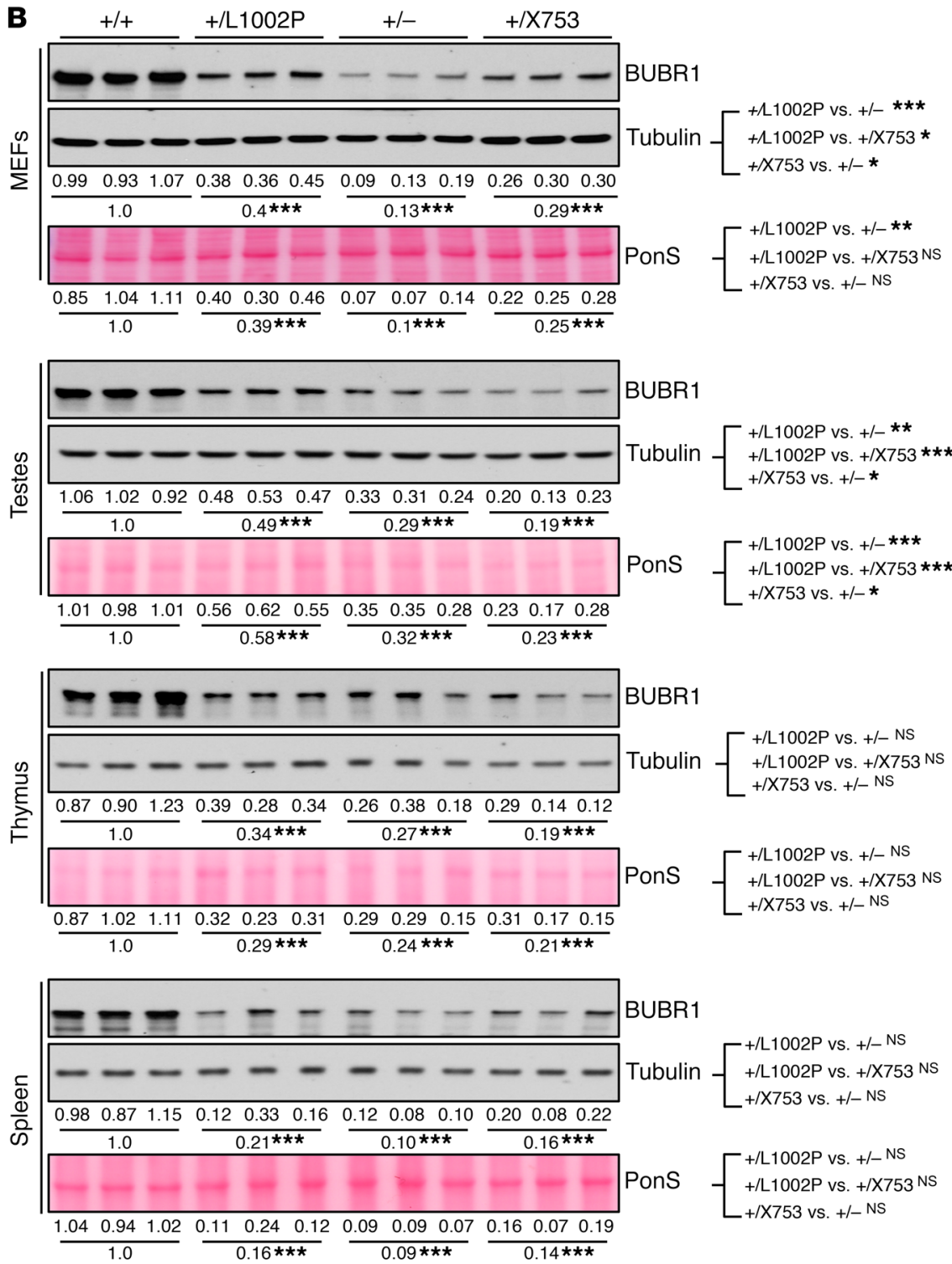

Figure 1. MVA alleles BUBR $1^{1012 \mathrm{P}}$ and $B U B R 1^{\mathrm{x} 753}$ yield low amounts of protein. (A) Schematic of BUBR1 protein. Locations of MVA patient mutations are indicated. Ken, Ken box; Tpr, tetratricopeptide repeat motif; D-box, destruction box; Glebs, Glebs-binding motif; Phe, Phe box; Kard, kinetochore attachment regulatory domain; Kinase, kinase domain. (B) Western blots of asynchronous passage- $5 \mathrm{MEF}$ and proliferative tissue lysates (6-week-old mice) probed for BUBR1. Total protein (Ponceau S [PonS] staining) and $\alpha$-tubulin served as independent loading controls. BUBR1 levels were quantified and normalized to $\alpha$-tubulin or PonS. Numerical values indicate quantified

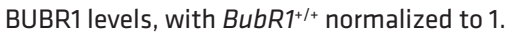
Values below the horizontal lines represent the mean. Statistical significance was determined using 1-way ANOVA with the Holm-Šídák post hoc test $(\mathbf{B}) .{ }^{*} P<0.05 ;{ }^{* *} P<0.01 ;{ }^{* *} P<0.001$. NS, not significant.
Models for heterozygous BUBR1 MVA mutations show phenotypic heterogeneity. Whether heterozygous carriers of BUBR1 MVA mutations might develop any symptoms associated with the syndrome is a key question that remains to be addressed. In a previous study, we examined mice that model an MVA allele carrier, heterozygous for the $B U B R 1^{\mathrm{X} 753}$ mutation, and found that these mice were indeed more susceptible to cancer and select progeroid phenotypes than their wild-type counterparts (21). To develop a more comprehensive understanding of the impact of heterozygous MVA mutations, we modeled heterozygous carriers of 2 other MVA alleles, $B U B R 1^{\mathrm{L} 1012 \mathrm{P}}$ and $B U B R 1^{-}$, the latter representing the null allele found in a biallelic MVA patient that also carries a $B U B R 1^{1909 \mathrm{~T}}$ mutation (2). The necessary cohorts of $B u b R 1^{+/+}, B u b R 1^{+/ L 1002 P}$, and $B u b R 1^{+/-}$mice were established by intercrossing $B u b R 1^{+/ L 1002 P}$ and
$B u b R 1^{+/-}$mice. Consistent with earlier data (21), $B u b R 1^{+/-}$mice had a modest, but significant, reduction in lifespan compared with $B u b R 1^{+/+}$mice (Figure 2A). BubR $1^{+/ L 1002 \mathrm{P}}$ mice also showed a strong trend toward reduced median lifespan that was close to reaching significance $(P=0.0516$, log-rank test). Although in both heterozygous mutant cohorts the incidence and spectrum of spontaneous tumors detectable at autopsy were similar to wild-type (Figure 2, B and C), tumor latencies in both mutants were significantly reduced (Figure 2A), indicating that both MVA mutations may promote tumorigenesis by accelerating tumor growth. Consistent with this, lymphomas from $B u b R 1^{+/ L 1002 \mathrm{P}}$ and $B u b R 1^{+/-}$mice, which developed with reduced latencies, contained significantly more mitotic cells than lymphomas of $B u b R 1^{+/+}$mice (Figure 2, D and E). A complementary experiment in which we analyzed tumor- 

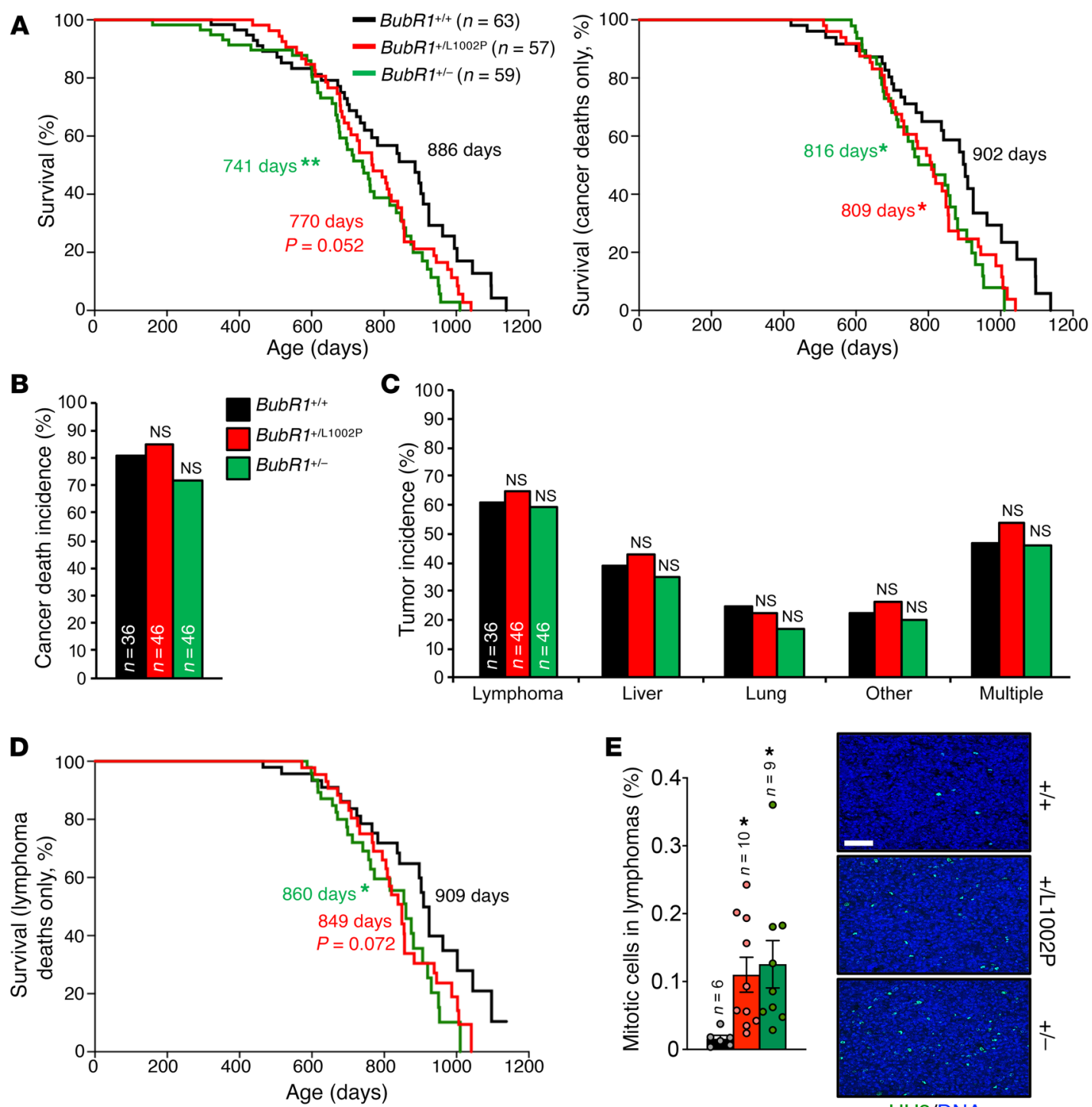

E

$\mathbf{F}$

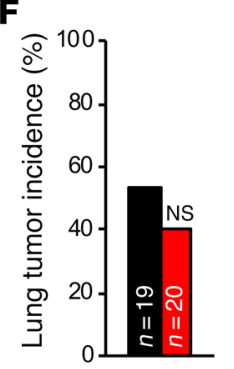

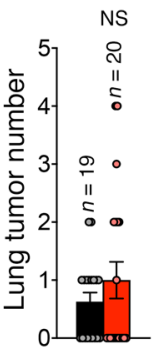

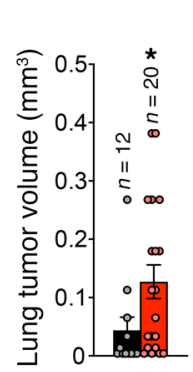

$\mathbf{G}$

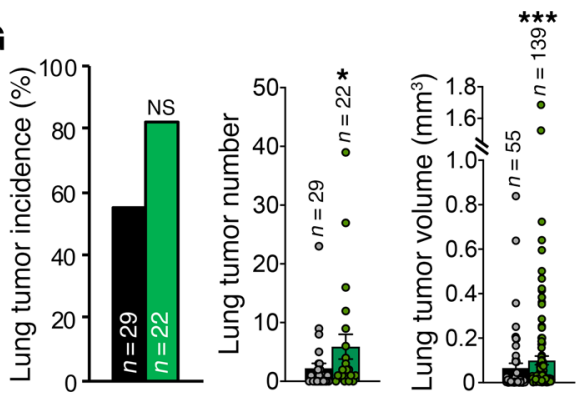

Figure 2. Carriers of single BUBR1 MVA mutations are phenotypically heterogeneous. (A) Kaplan-Meier curves showing overall survival (left) and cancer deaths only (right) of the indicated mice. Values associated with curves denote median survival. (B) Incidence of cancer deaths in the indicated mice. (C) Spectrum of cancer types associated with cancer deaths in the indicated mice. (D) Kaplan-Meier curve for lymphoma deaths of the indicated mice. Median survival is indicated. (E) Mitotic index of the lymphoma tumor samples collected from the indicated moribund mice. Images of representative pHH3labeled lymphoma sections are shown. Scale bar: $50 \mu \mathrm{m}$. (F and $\mathbf{G})$ DMBA-induced lung tumor incidence, multiplicity, and volume in the indicated mice. Bars in E-G represent the mean \pm SEM, and dots represent individual samples. Each $n$ for all experiments represents individual mice, with the exception of tumor volume in $\mathbf{F}-\mathbf{G}$ where individual tumors are represented. Statistical significance was determined using a log-rank test (A and $\mathbf{D}), 2$-tailed Fisher's exact test (B-C and $\mathbf{F}-\mathbf{G}$, incidence), 1-way ANOVA with the Holm-Šídák post hoc test (E), and a Mann-Whitney $U$ test (F-G, tumor number and volume). ${ }^{*} P<0.05 ;{ }^{* *} P<0.01 ;{ }^{* *} P<0.001$. NS, not significant. 

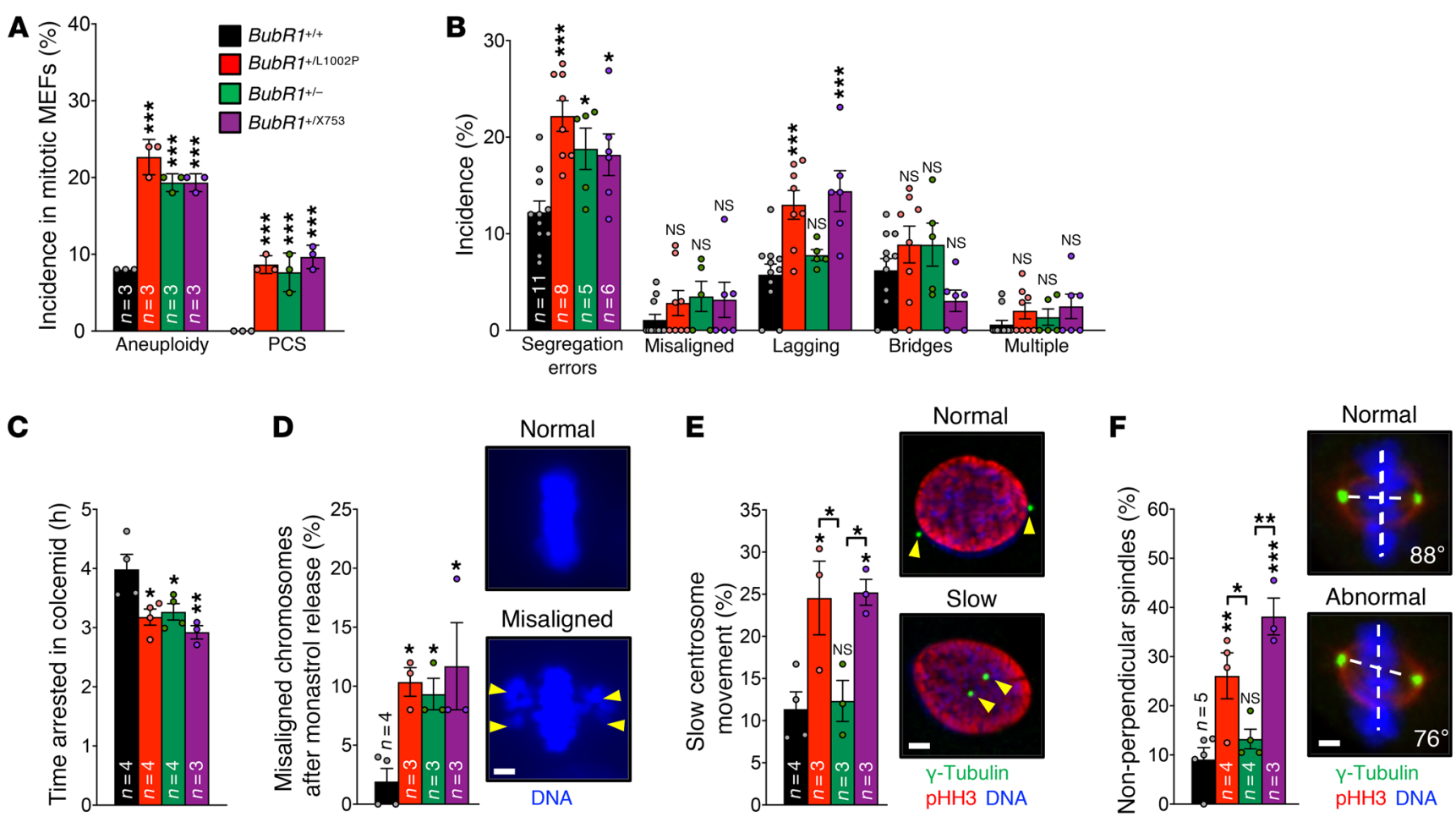

Figure 3. Surveillance mechanisms that ensure high-fidelity chromosome segregation are defective in monoallelic BubR1 MVA mutant MEFs. (A) Incidence of aneuploidy and PCS in mitotically arrested passage- 5 MEFs. (B) Chromosome segregation errors in passage- 5 MEFs assessed by live-cell imaging of MEFs expressing H2B-mRFP. Each $n$ indicates independent MEF lines for all genotypes, except for $B u b R 7^{+/ L 1002 P}$ and $B u b R 1^{+/ X 753}$, where technical replicates were performed on 4 and 3 independent lines, respectively. (C) Colcemid-challenge assay on passage-5 MEFs measuring SAC activity. (D) Error correction in the indicated passage- 5 MEFs assessed by monastrol washout assay. Representative images are shown. Yellow arrowheads indicate misaligned chromosomes. (E and $\mathbf{F}$ ) Incidence of slow centrosome movement $(\mathbf{E})$ and non-perpendicular spindles $(\mathbf{F})$ in the indicated passage-5 MEFs. Representative images are shown. Yellow arrowheads indicate the location of the centrosomes. Each $n$ indicates independent MEF lines, unless otherwise noted. Data are presented as mean \pm SD $(\mathbf{A})$ and mean \pm SEM (B-F), and dots represent individual samples. Scale bars: $3 \mu \mathrm{m}(\mathbf{D}$ and $\mathbf{E})$ and $2 \mu \mathrm{m}(\mathbf{F})$. Statistical significance was determined using 1-way ANOVA with the Holm-Šídák post hoc test. ${ }^{*} P<0.05 ;{ }^{* *} P<0.01$; ${ }^{* *} P<0.001$. NS, not significant.

igenesis by performing a timed sacrifice at $18-20$ months of age confirmed that the incidence of tumor formation and the spectrum of tumors in $B u b R 1^{+/ L 1002 P}$ and $B u b R 1^{+/}$mice were unchanged (Supplemental Figure 3, A and B).

To extend our cancer susceptibility studies, we challenged $B u b R 1^{+/+}, B u b R 1^{+/ 11002 P}$, and $B u b R 1^{+/}$mice with 7,12-dimethylbenz(a) anthracene (DMBA), a carcinogen that primarily induces lung tumors when applied on the dorsal skin at postnatal day 5 (22). Both $B u b R 1^{+/ L 1002 \mathrm{P}}$ and $B u b R 1^{+/-}$mice showed no increase in lung tumor incidence in this tumor bioassay. Lung tumor multiplicity and size, however, were significantly increased in $B u b R 1^{+/-}$mice, and likewise lung tumor size was also increased in $B u b R 1^{+/ L 1002 P}$ mice, indicative of accelerated tumor growth and/or initiation (Figure 2, F and G). Thus, together with earlier studies in $B u b R 1^{+/ X 753}$ mice, these findings imply that heterozygous carriers of BUBR1 MVA mutations may be at increased risk of tumor formation, albeit to different degrees.

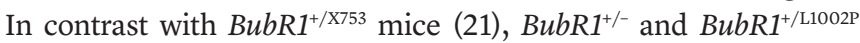
mice showed little to no evidence of accelerated aging phenotypes (Supplemental Figure 4, A-E).

Heterozygous BUBR1 MVA mutants demonstrate intricate mitotic phenotypes. To examine the impact of heterozygous BUBR1 MVA mutations on chromosome number integrity, we performed chromosome counts on metaphase spreads of MEFs at passage-5. Aneuploidy was markedly elevated in $B u b R 1^{+/ L 1002 P}, B u b R 1^{+/}$, and
BubR $1^{+/ X 753}$ MEFs compared with BubR1 $1^{+/+}$MEFs (Figure 3A and Supplemental Table 2). Premature chromatid separation (PCS), a hallmark of MVA patients, was observed in all 3 MVA allele carriers (Figure 3A). We complemented these experiments with interphase fluorescence in situ hybridization (FISH) analyses for chromosomes 4 and 7 in these MEFs, which targets the entire cell population rather than only the mitotically active fraction. Aneuploidy rates were again similarly increased in all 3 MVA allele carriers (Supplemental Figure 5 and Supplemental Table 3).

By monitoring chromosome movements during mitosis in live cells expressing histone $\mathrm{H} 2 \mathrm{~B}$-monomeric red fluorescent protein (H2B-mRFP), we observed small, but significant increases in chromosome segregation errors for all 3 heterozygous BubR1 MVA mutants (Figure 3B). In all 3 heterozygous mutants both the SAC and attachment error correction machinery were impaired, providing an explanation for the observed increase in segregation errors (Figure 3, C and D). However, both high-fidelity chromosome segregation insurance systems were similarly impaired in all 3 mutants, prompting us to screen for additional mitotic defects that might explain the higher rates of lagging chromosomes in $B u b R 1^{+/ 11002 P}$ and $B u b R 1^{+/ / 7753} \mathrm{MEFs}$ (Figure 3B). Lagging chromosomes are frequently caused by aberrancies in centrosome disjunction or movement that result in the formation of non-perpendicular spindles that are enriched in merotelic kinetochore-microtubule attachments 
A

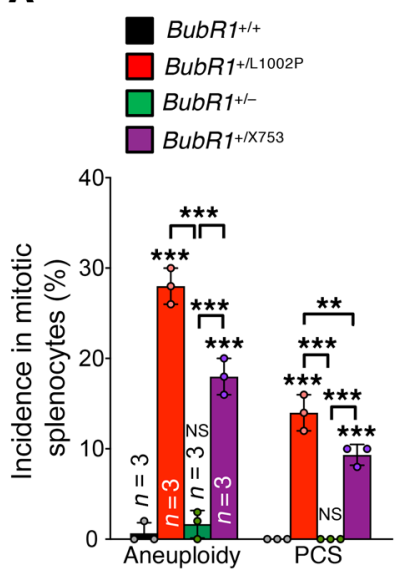

B

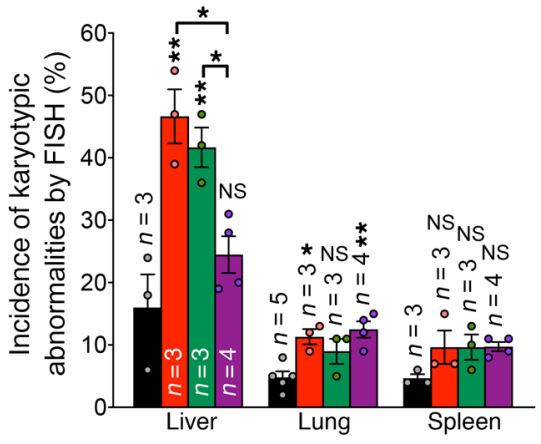

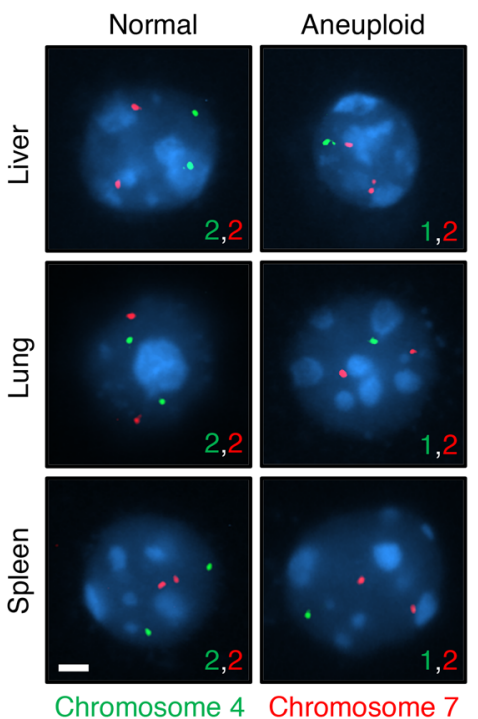

Figure 4. Monoallelic BubR1 MVA mutants exhibit mosaic aneuploidies. (A) Incidence of aneuploidy and PCS in mitotic splenocytes of 5-month-old mice. (B) Incidence of aneuploidy in tissues from 22- to 24-month-old mice assessed by FISH for chromosomes 4 and 7. Representative images are shown. Each $n$ indicates cells or tissues from independent mice. Data are presented as mean \pm SD (A) and mean \pm SEM (B), and dots represent individual samples. Scale bar: $2 \mu \mathrm{m}$ (B). Statistical significance was determined using 1-way ANOVA with the Holm-Šídák post hoc test. ${ }^{*} P<0.05$; ${ }^{* *} P<0.01$; ${ }^{* *} P<0.001$. NS, not significant.

$(23,24)$. Indeed, both monoallelic MVA mutants with increased chromosome lagging formed non-perpendicular spindles at elevated rates, due to slow centrosome movement (Figure 3, $\mathrm{E}$ and $\mathrm{F}$ ). Although it remains to be determined how BUBR1 impacts centrosome movement, it is clear that there is an allele-dependent effect on this process. Overall, the above experiments indicate that allelic effects beyond BUBR1 levels alone contribute to the mitotic phenotypes of monoallelic MVA mutants.

We complemented our analyses of cultured MEFs with

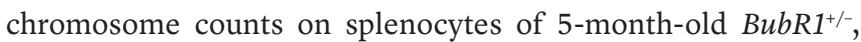
$B u b R 1^{+/ L 1002 P}$, and $B u b R 1^{+/ X 753}$ mice. Specifically, we briefly cultured freshly harvested splenocytes in the presence of colcemid for 4 hours prior to preparing metaphase spreads and counting chromosomes. This method assesses the percentage of aneuploid cells among the subset of cycling splenocytes entering mitosis. We observed major differences in aneuploidy between heterozygous BubR1 MVA mutants, with $2 \%, 18 \%$, and $28 \%$ of mitotic splenocytes showing abnormal chromosome numbers in $B u b R 1^{+/-}, B u b R 1^{+/ X 753}$, and $B u b R 1^{+/ L 1002 \mathrm{P}}$ mice, respectively (Figure $4 \mathrm{~A}$ and Supplemental Table 4). The same was true for PCS (Figure 4A). To investigate karyotypic instability in greater depth, we conducted interphase FISH analysis for chromosomes 4 and 7 on liver, lung, and spleen of $B u b R 1^{+/+}, B u b R 1^{+/-}, B u b R 1^{+/ L 1002 P}$, and $B u b R 1^{+/ X 753}$ mice ranging in age from $22-24$ months. Instead of tissue sections, we prepared single-cell suspensions from the above tissues and dropped these on slides to avoid the loss of nuclear content that occurs with sectioning (14). Our analyses showed that aneuploidy was increased in livers of $B u b R 1^{+/ L 1002 P}$ and $B u b R 1^{+/-}$mice, as well as in lungs of $B u b R 1^{+/ L 1002 \mathrm{P}}$ and $B u b R 1^{+/ X 753}$ mice (Figure 4B and Supplemental Table 3). Aneuploidy rates in the spleen, as measured by FISH, were not elevated in any of the monoallelic MVA mutants, even though substantial increases were observed in mitotically active splenocytes of these strains
(Figure 4, A and B). One possible explanation might be that chromosome missegregation or aneuploid cell survival rates differentially change over time for each of the mutants. Notwithstanding these differences, collectively, these data suggest that a key feature of MVA syndrome patients, increased aneuploidy in multiple tissues, is conserved in heterozygous carriers of BUBR1 MVA mutations.

Progeroid $B u b R 1^{+/ X 753}$ mice exhibit hyperactive mTORC1 signaling. To further investigate the basis for the phenotypic heterogeneity among monoallelic MVA mutants, we conducted genome-wide transcriptome profiling on gastrocnemius muscle from 3-month-old $B u b R 1^{+/ X 753}, B u b R 1^{+/ L 1002 P}$, and $B u b R 1^{+/+}$mice. This tissue was chosen because in $B u b R 1^{+/ X 753}$ mice it is selectively subject to accelerated aging (21). The 3-month time point was selected because at this age no signs of muscle aging are detected, allowing for detection of primary alterations resulting from the presence of the $B u b R 1^{\mathrm{X} 753}$ allele rather than secondary transcriptional changes associated with sarcopenia. Strikingly, several hundred differentially expressed genes (DEGs) emerged when comparing $B u b R 1^{+/ X 753}$ with $B u b R 1^{+/+}$, whereas the transcriptome of $B u b R 1^{+/ L 1002 P}$ skeletal muscle was similar to that of $B u b R 1^{+/+}$, yielding only 3 DEGs (Figure 5A). Comparison of $B u b R 1^{+/ X 753}$ with $B u b R 1^{+/ L 1002 P}$ also yielded a significant number of DEGs, albeit fewer than compared with $\mathrm{BubR}^{+/+}$(Figure 5A). Functional enrichment analyses with the DEGs using the STRING database (25) revealed that the majority of cellular functions that were significantly enriched in the upregulated DEGs of $B u b R 1^{+/ X 753}$ skeletal muscle were linked to the mTORC1 signaling pathway (Figure $5 \mathrm{~B}$ ), which has been linked to aging (26). In addition, a high percentage of the upregulated DEGs from the $B u b R 1^{+/ X 753}$ versus $B u b R 1^{+/ L 1002 P}$ comparison function in mTORC1-related biological processes (Supplemental Figure 6, A and B). Consistent with this, phosphorylation of 2 key mTORC1 substrates, p70 S6 kinase and $4 \mathrm{EBP} 1$, were markedly increased in skeletal muscle of 3-month- 
A

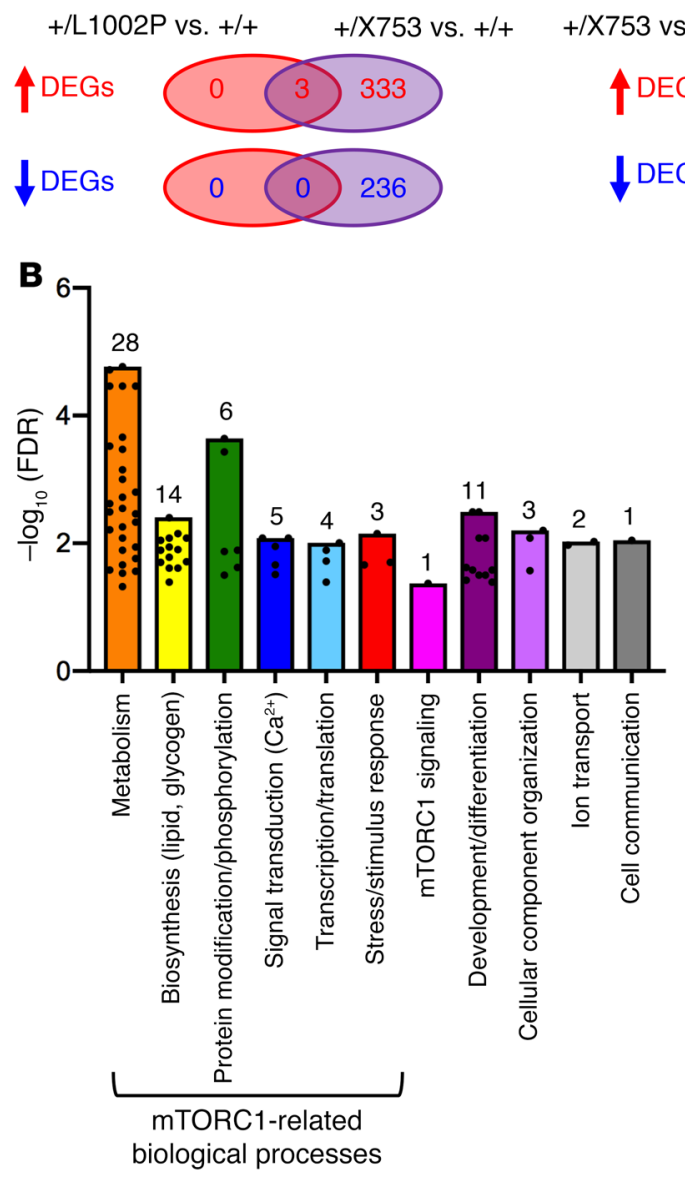

C Skeletal muscle

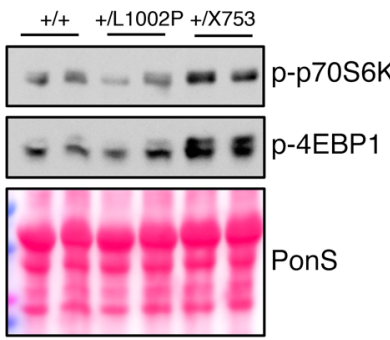

D
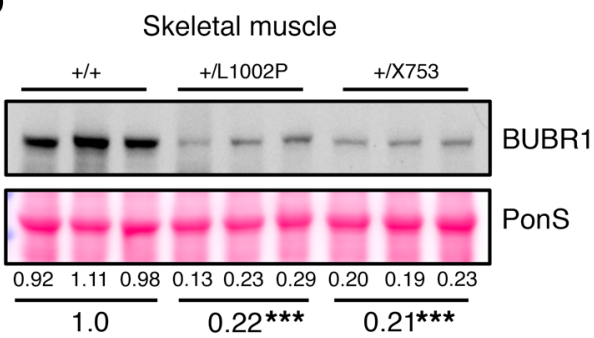

+/L1002P vs. $+/ X 753$ NS

Figure 5. Progeroid BubR1+/x753 mice exhibit aberrant cellular signaling. (A) Venn diagrams of DEGs from RNA sequencing analyses of gastrocnemius muscle from 3-month-old mice of the indicated genotypes. $\uparrow$, upregulated genes; $\downarrow$, downregulated genes. $n=3$ independent mice/genotype. (B) Func-

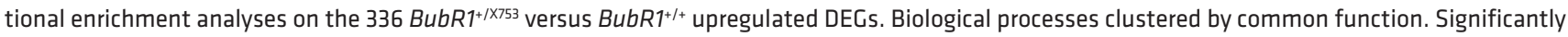
enriched (FDR $<0.05,-\log _{10}>1.3$ ) processes are shown. Bars represent maximum - $\log _{10}$ (FDR) per functional group, and dots represent individual annotations for pathways under a given functional group. Numbers above bars represent the total number of pathways per group. (C) Western blot of gastrocnemius muscle from two 3-month-old mice of the indicated genotypes. PonS served as the loading control. (D) Western blot of gastrocnemius muscle from three 10-day-old mice of the indicated genotypes, probed for BUBR1. PonS served as the loading control. BUBR1 levels were quantified and are shown as in Figure 1B. See Methods for statistical analyses for RNA sequencing and functional enrichment analyses (A and B). Statistical significance was determined using 1-way ANOVA with the Holm-Šídák post hoc test (D). ${ }^{* *} P<0.001$. NS, not significant.

old $B u b R 1^{+/ X 753}$ mice compared with corresponding lysates from $B u b R 1^{+/ L 1002 P}$ and $B u b R 1^{+/+}$mice, as determined by Western blot

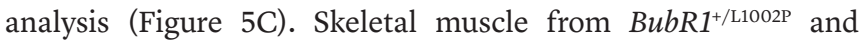
$B u b R 1^{+/ X 753}$ mice had similarly reduced BUBR1 protein levels compared with $\mathrm{BubR}^{+/+}$mice, indicating that differences in BUBR1 protein levels are unlikely to account for the differential impact of these MVA mutations on mTORC1 signaling (Figure 5D). The possibility that BUBR1 levels initially decline similarly in BubR $1^{+/ L 1002 P}$ and $B u b R 1^{+/ X 753}$ young adult mouse tissues, but then more rapidly in $B u b R 1^{+/ / 7753}$ mice as animals reach a more advanced age is also unlikely, as suggested by Western blot analysis for BUBR1 on spleen lysates of 24-month-old mice (Supplemental Figure 7).

BubR $1^{H / L 1002 P}$ mice are viable and model MVA syndrome-associated pathologies. Following our unsuccessful attempt to model the $B U B R 1^{\mathrm{X} 753 / 1012 \mathrm{P}}$ MVA patient in mice, we asked whether the $B u b R 1^{\mathrm{H}}$ allele, which mimics the hypomorphic allele found in patients with monoallelic BUBR1 mutations $(1,8)$, might produce viable offspring when combined with $B u b R 1^{\mathrm{x} 753}$ or $B u b R 1^{1 \mathrm{~L} 1002 P}$. Indeed, intercrosses of $B u b R 1^{+/ X 753}$ and $B u b R 1^{+/ H}$ mice yielded viable $B u b R 1^{\mathrm{H} / \mathrm{X} 753}$ offspring; however, these mice failed to thrive and died within 18 hours after birth (Figure 6A), reminiscent of $B u b R 1^{-/ H}$ mice (8). Comparative Western blot analysis of MEF lysates revealed that $B u b R 1^{-/ H}$ and $B u b R 1^{\mathrm{H} / \mathrm{X} 753} \mathrm{MEFs}$ had a similar reduction in BUBR1 protein (Figure 6B). On the other hand, intercrosses of $B u b R 1^{+/ L 1002 P}$ and $B u b R 1^{+/ H}$ mice yielded $B u b R 1^{\mathrm{H} / L 1002 \mathrm{P}}$ mice that had a normal appearance at birth, but became growth retarded during postnatal development, although not as severely as $B u b R 1^{\mathrm{H} / \mathrm{H}}$ mice (Figure 6, C and D). Although postnatal viability of $B u b R 1^{\mathrm{H} / X 753}$ and $B u b R 1^{\mathrm{H} / \mathrm{L1002P}}$ mice was markedly different, residual BUBR1 protein levels in MEFs of these genotypes were not significantly different from each other (Supplemental Figure 8A). Furthermore, despite the significant differences in body size between $B u b R 1^{\mathrm{H} / \mathrm{L1002 \textrm {P }}}$ and $B u b R 1^{\mathrm{H} / \mathrm{H}}$ mice, the level of BUBR1 protein reduction in MEFs and multiple tissues appeared to be very similar between the mutants, with significant 
A
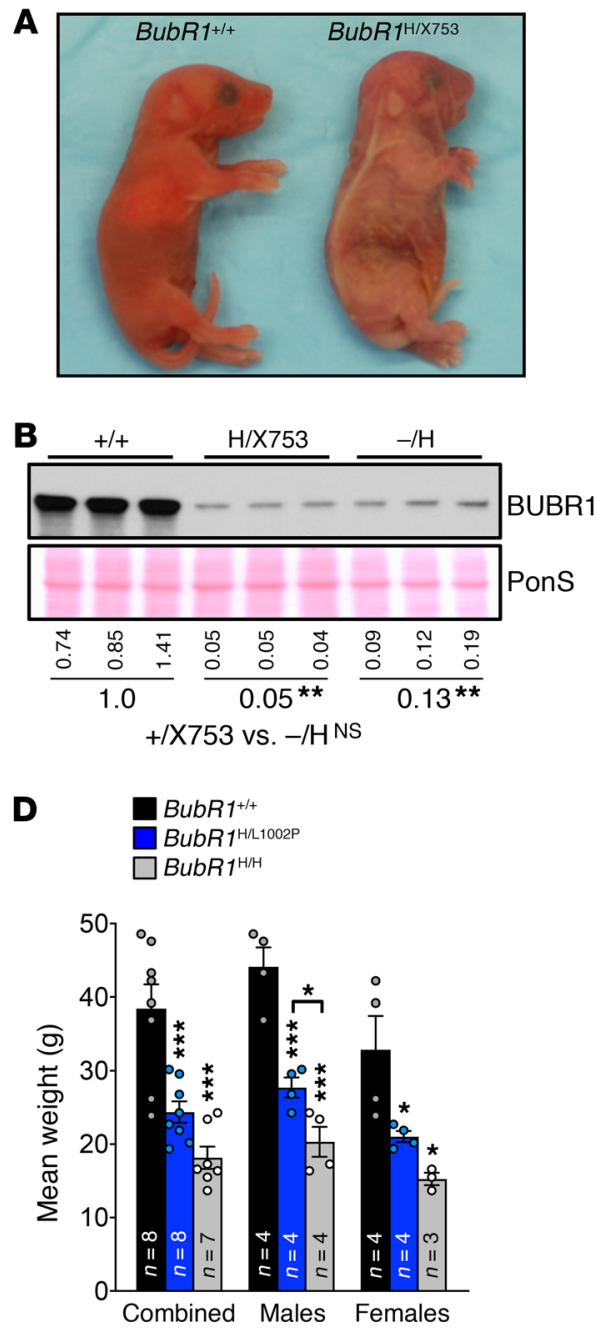
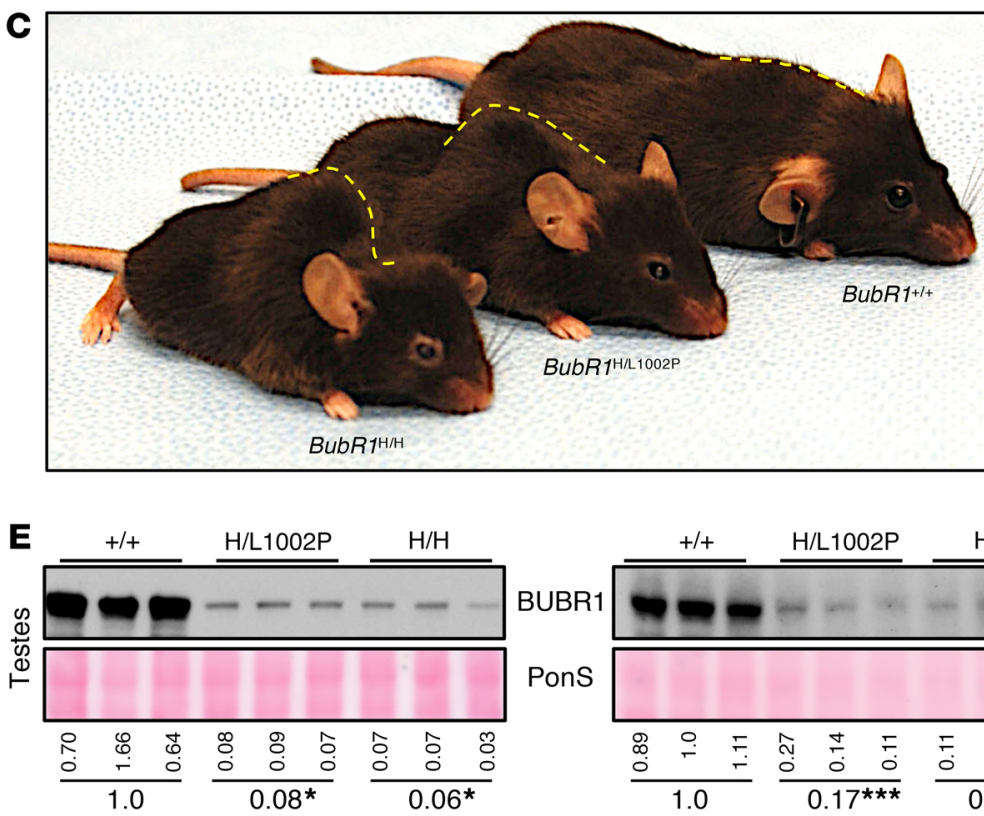

$\mathrm{H} / \mathrm{L} 1002 \mathrm{P}$ vs. $\mathrm{H} / \mathrm{H}^{\mathrm{NS}}$

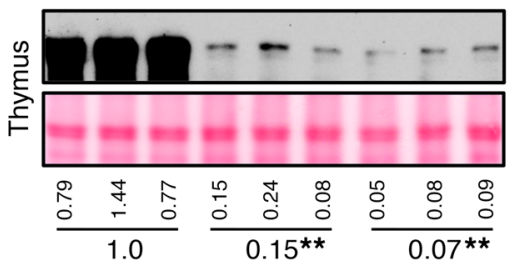

$\mathrm{H} / \mathrm{L} 1002 \mathrm{P}$ vs. $\mathrm{H} / \mathrm{H}$ NS

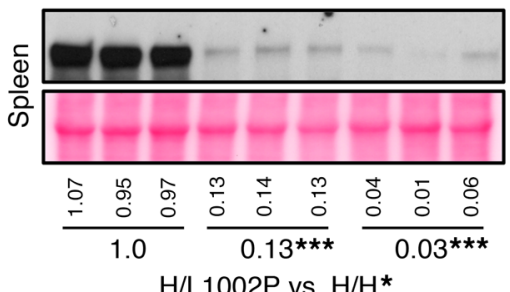

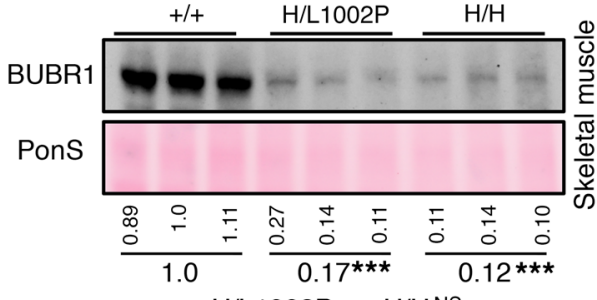

$\mathrm{H} / \mathrm{L} 1002 \mathrm{P}$ vs. $\mathrm{H} / \mathrm{H}^{\mathrm{NS}}$
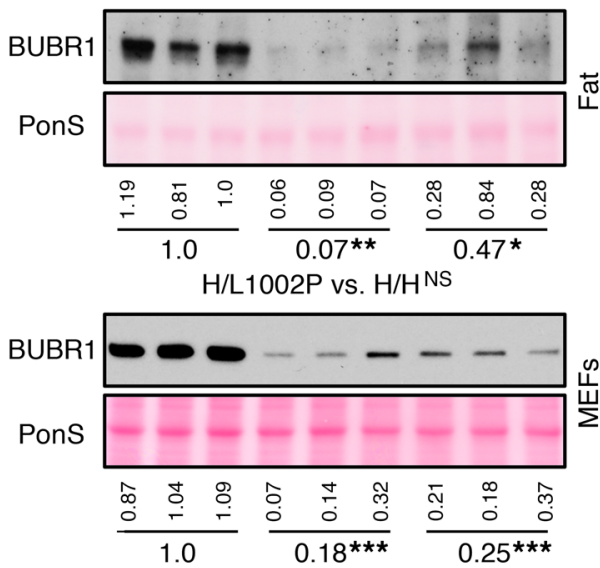

$\mathrm{H} / \mathrm{L} 1002 \mathrm{P}$ vs. $\mathrm{H} / \mathrm{H}^{\mathrm{NS}}$
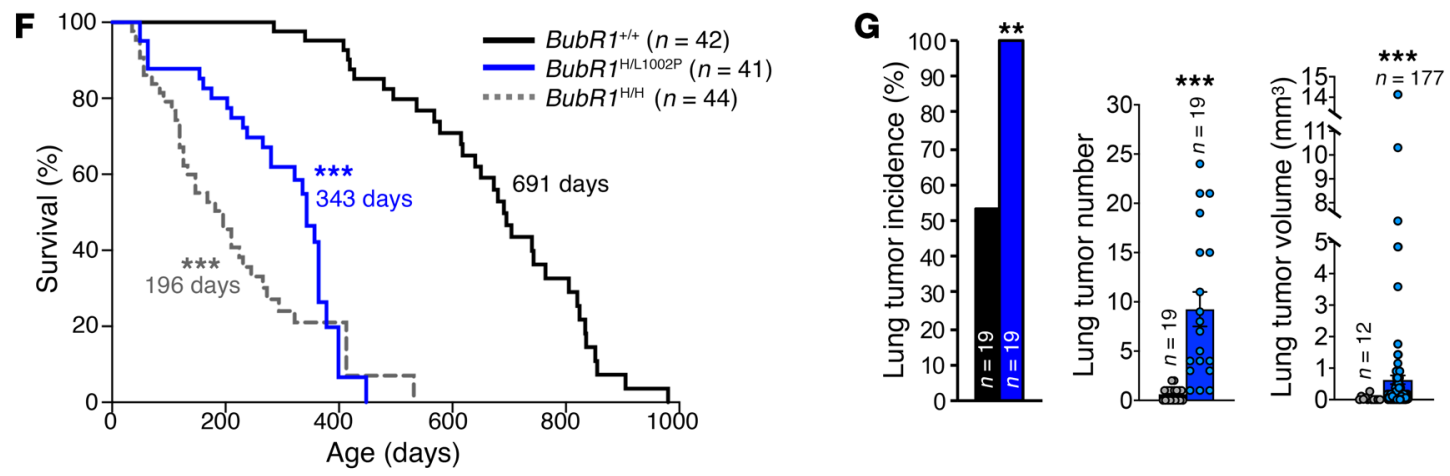

Figure 6. Mouse models carrying 2 allelic BubR1 MVA patient variants are viable. (A) Image of $B u b R 1^{+/+}$and $B u b R 1^{H / x 753}$ pups a few hours after birth. (B) Western blots of asynchronous passage-5 MEF lysates, probed for BUBR1. PonS-stained proteins served as the loading control. Three independent lines were used for analyses. BUBR1 levels were quantified and are shown as in Figure 1B. (C) Representative image of 8- to 10-month-old mice. Dashed

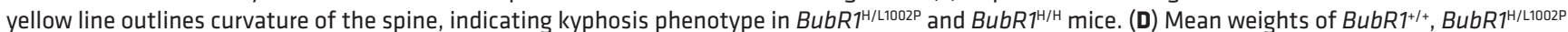
and $B u b R 7^{H / H}$ mice at 5-7 months of age. Each $n$ indicates independent mice. (E) Western blots of tissues from 6-week-old (testes, thymus, and spleen) and 10-day-old (gastrocnemius muscle and fat) mice, and passage-5 asynchronous MEFs of the indicated genotypes. PonS served as the loading control. BUBR1 levels were quantified and are shown as in Figure 1B. (F) Survival curves for the indicated mouse models. Values associated with curves denote median survival. $P<0.05$ for $B u b R 1^{H / H}$ versus $B u b R 1^{H / L 1002 P}$. (C) DMBA-induced lung tumor incidence, multiplicity, and volume in the indicated mice. Data in $\mathbf{D}$ and $\mathbf{G}$ are presented as the mean \pm SEM, and dots represent individual samples. Statistical significance was determined using 1-way ANOVA with the Holm-Šíák post hoc test (B, D, and E), log-rank test (F), 2-tailed Fisher's exact test (G, incidence), and Mann-Whitney $U$ test (D and $\mathbf{G}$, tumor number and volume). ${ }^{*} P<0.05 ;{ }^{* *} P<0.01 ;{ }^{* *} P<0.001$. NS, not significant. 

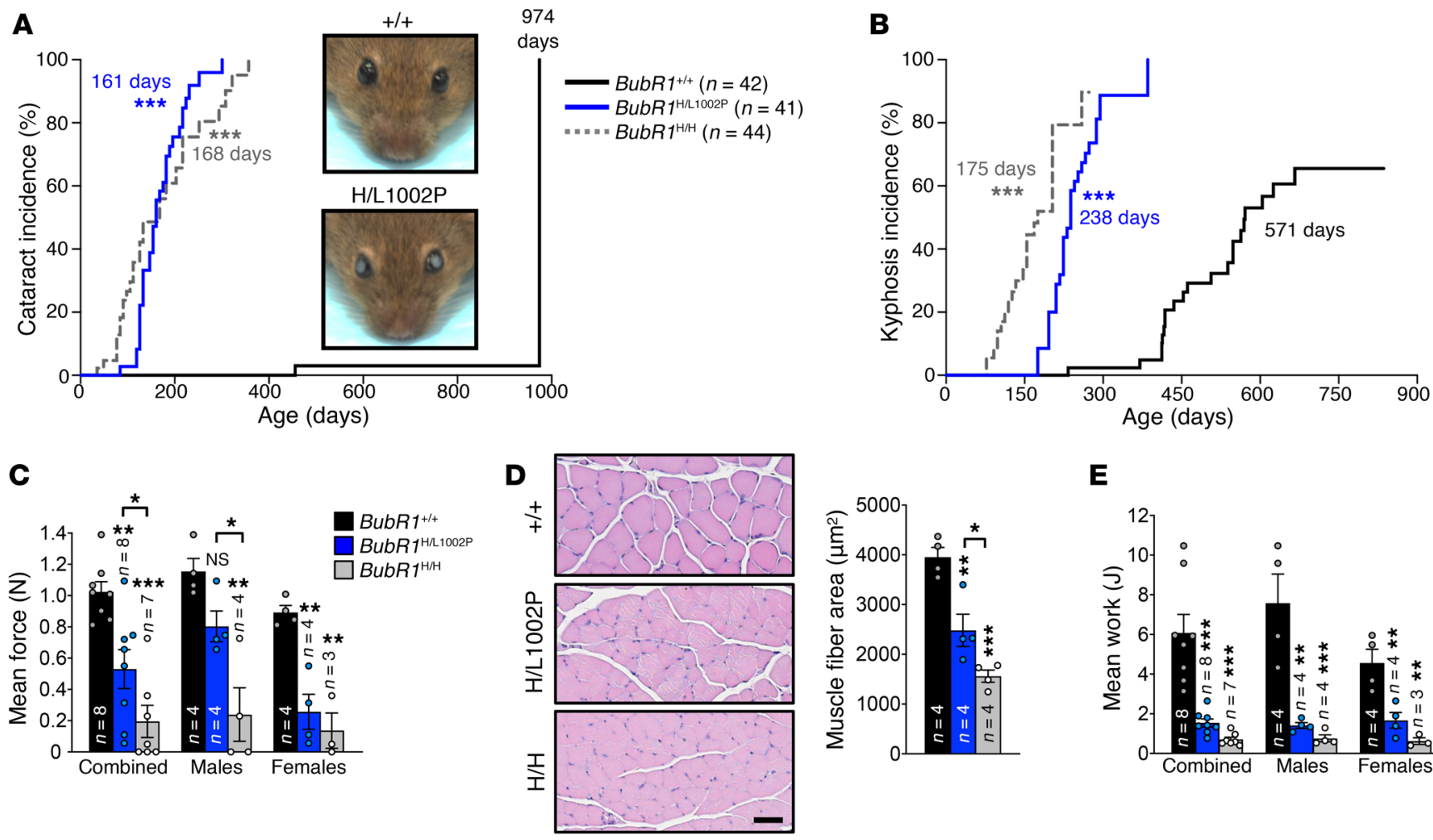

$\mathbf{E}$
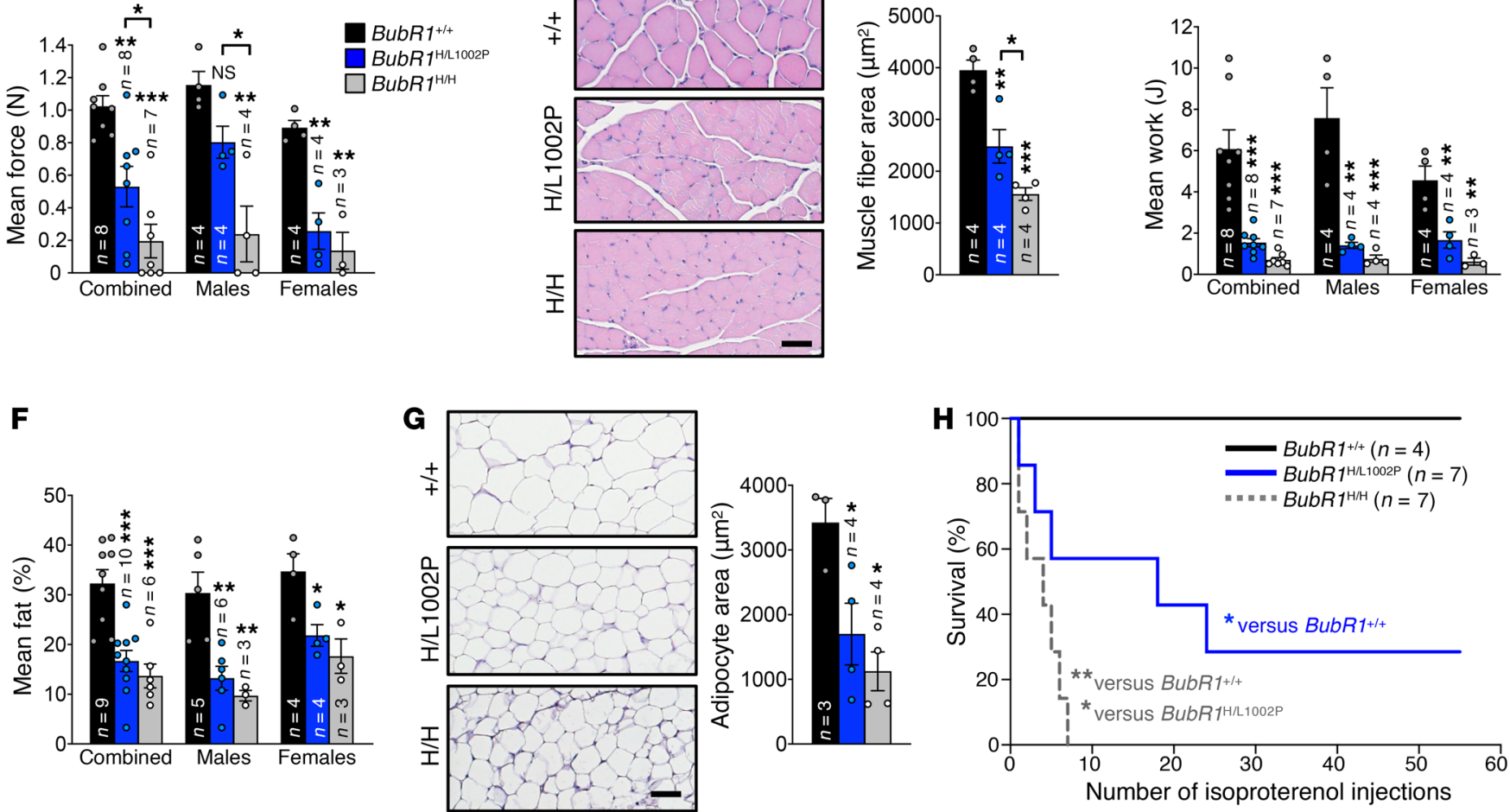

Figure 7. BubR $1^{1 / L 002 P}$ and $B u b R 1^{H / H}$ mice recapitulate MVA syndrome heterogeneity. (A) Kaplan-Meier curves of cataract onset in the indicated mice. Values associated with curves denote median onset. Representative images of 5-month-old mice are shown in insets. (B) Kaplan-Meier curves of kyphosis onset in the indicated mice. Values associated with curves denote median onset. $P<0.001$ for BubR $1^{H / H}$ versus BubR $1^{H / L 1002 P}$. (C) Analysis of forelimb grip strength in the indicated 5- to 7-month-old mice. (D) Histological analyses of gastrocnemius and measurement of muscle fiber cross-sectional area in the indicated 8- to 10-month-old mice. Representative images shown. Scale bar: $50 \mu \mathrm{m}$. (E) Mean work output, in joules (J), during a treadmill exercise test of 5- to 7-month-old mice. (F) Mean total body fat percentage of 8- to 10-month-old mice, as determined by echo-MRI analyses. (G) Histological analyses of inguinal adipose tissue (IAT) and measurement of adipocyte cross-sectional area in the indicated 8- to 10-month-old mice. Representative images shown. Scale bar: $50 \mu \mathrm{m}$. (H) Survival curves of the indicated 4- to 7-month-old mice exposed to a low-dose isoproterenol regimen to evaluate cardiac stress tolerance. Each $n$ indicates independent mice for all experiments. Data in C-G are presented as mean \pm SEM, and dots represent individual samples. Statistical significance was determined using a log-rank test $(\mathbf{A}, \mathbf{B}$, and $\mathbf{H})$ and 1-way ANOVA with the Holm-Šídák post hoc test $(\mathbf{C}-\mathbf{G}) .{ }^{*} P<0.05$; ${ }^{*} P<0.01$; ${ }^{* * *} P<0.001$. NS, not significant.

differences observed only in the spleen (Figure 6E). BubR $1^{\mathrm{H} / 1002 \mathrm{P}}$ mice had a median lifespan of 343 days compared with 691 days for $B u b R 1^{+/+}$mice (Figure $6 \mathrm{~F}$ ). Although BubR $1^{\mathrm{H} / \mathrm{L1002P}}$ mice were shortlived, on average they lived significantly longer than $B u b R 1^{\mathrm{H} / \mathrm{H}}$ mice, which had a median lifespan of 196 days (Figure 6F).

Similar to $B u b R 1^{\mathrm{H} / \mathrm{H}}$ mice, $B u b R 1^{\mathrm{H} / \mathrm{L1} 1002 \mathrm{P}}$ mice were not prone to spontaneous tumors, with only $13 \%$ of mice dying with macroscopically detectable tumors. Furthermore, the incidence and multiplicity of DMBA-induced lung tumors were significantly elevated (Figure 6G), analogous to BubR $1^{\mathrm{H} / \mathrm{H}}$ mice (12). However, the average lung tumor size was increased in DMBA-treated

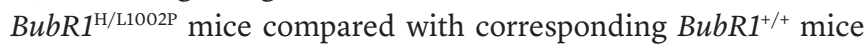
(Figure 6G). This was not observed in $B u b R 1^{\mathrm{H} / \mathrm{H}}$ mice (12) and is perhaps a feature of select MVA alleles, as this was also noted in a milder form in $B u b R 1^{+/ L 1002 P}, B u b R 1^{+/-}$, and $B u b R 1^{1^{+/ X 753}}$ mice (Figure 2, F and G, and ref. 21). 
BubR1 $1^{H / L 1002 P}$ and BubR1 $1^{H / H}$ mice model MVA syndrome heterogeneity. We sought to further explore the extent to which $B u b R 1^{\mathrm{H} / \mathrm{L} 1002 \mathrm{P}}$ and $\mathrm{BubR} 1^{\mathrm{H} / \mathrm{H}}$ mice are subject to phenotypic heterogeneity, a hallmark of MVA syndrome. To this end, we focused on a series of progeroid phenotypes that characterize $B u b R 1^{\mathrm{H} / \mathrm{H}}$ mice, most of which represent MVA-associated pathologies. We discovered that $B u b R 1^{\mathrm{H} / \mathrm{L} 1002 \mathrm{P}}$ mice are highly sensitive to cataract formation, the median onset of which was 161 days, which is nearly identical to that of $B u b R 1^{\mathrm{H} / \mathrm{H}}$ mice (median onset, 168 days; Figure $7 \mathrm{~A}$ ).

A second overt progeroid phenotype of $B u b R 1^{\mathrm{H} / \mathrm{H}}$ mice, kyphosis, also developed in $B u b R 1^{\mathrm{H} / \mathrm{L} 1002 \mathrm{P}}$ mice, but with delayed latency compared with $B u b R 1^{\mathrm{H} / \mathrm{H}}$ mice (median onset, 238 days versus 175 days; Figure 7B). In $B u b R 1^{\mathrm{H} / \mathrm{H}}$ mice, this phenotype is linked to a number of features of sarcopenia, including reduced grip strength, muscle fiber atrophy, and reduced work output during treadmill exercise (Figure 7, C-E, and Supplemental Figure $8, \mathrm{~B}$ and C). Importantly, these same features were observed in $B u b R 1^{\mathrm{H} / \mathrm{L} 1002 \mathrm{P}}$ mice, although typically they were less severe in $B u b R 1^{\mathrm{H} / \mathrm{L} 1002 \mathrm{P}}$ mice compared with $B u b R 1^{\mathrm{H} / \mathrm{H}}$ mice, which is in accordance with the delay in kyphosis onset.

Another key progeroid phenotype of $B u b R 1^{\mathrm{H} / \mathrm{H}}$ mice is fat tissue atrophy (lipodystrophy). Echo-MRI analysis on 8- to 10-monthold mice indicated that this phenotype was fully recapitulated in $B u b R 1^{\mathrm{H} / \mathrm{L} 1002 \mathrm{P}}$ mice (Figure $7 \mathrm{~F}$ ), which we confirmed by subsequent weighing of individual subcutaneous and visceral fat depots (Supplemental Figure 8D). Furthermore, the average size of fat cells was markedly reduced in $B u b R 1^{\mathrm{H} / \mathrm{L} 1002 \mathrm{P}}$ mice (Figure $7 \mathrm{G}$ ). The extent of the size reduction was similar to $B u b R 1^{\mathrm{H} / \mathrm{H}}$ mice.

Loss of cardiac stress tolerance is a hallmark of aging and has previously been linked to decreasing BUBR1 levels with aging (14). In accordance with this, cardiac stress tolerance of $B u b R 1^{\mathrm{H} / \mathrm{H}}$ mice is very low and is thought to be the primary cause of premature death in this model. To determine whether cardiac stress tolerance might be reduced in $B u b R 1^{\mathrm{H} / \mathrm{L} 1002 \mathrm{P}}$ mice, and if so, whether the magnitude of the decline might correlate with the extent of lifespan shortening, we performed an isoproterenol challenge test. In this assay, which does not impact survival of $B u b R 1^{+/+}$mice, we intraperitoneally injected a low dose of the $\beta$-adrenergic drug isoproterenol twice a day for 4 weeks (13). As expected, $B u b R 1^{\mathrm{H} / \mathrm{H}}$ mice were highly sensitive to repeated isoproterenol administration, with half of the animals dying within 5 injections (Figure $7 \mathrm{H}$ ). Lethality was also observed in $B u b R 1^{\mathrm{H} / \mathrm{L} 1002 \mathrm{P}}$ mice, but to a lesser extent, as $50 \%$ of the animals expired after 18 injections and some of the remaining mice showed no overt susceptibility. These data strengthen the idea that reduced tolerance to cardiac stress is a key determinant of lifespan shortening in biallelic BubR1 MVA syndrome models. Collectively, these data indicate that phenotypic heterogeneity occurs despite similarity in overall BUBR1 protein level and that some phenotypes are more prone to divergency than others.

Progeroid heterogeneity occurs despite mitotic phenotype similarity. The random reshuffling of chromosomes in multiple tissues is a common feature of MVA syndrome patients, irrespective of whether they have mutations in BUBR1, CEP57, or TRIP13 (1-4). However, whether and how the clinical heterogeneity that characterizes MVA syndrome might be driven by heterogeneity of mitotic defects has been difficult to assess, primarily because of the rarity of the syndrome and the limited availability of patient sam- ples. Although attempts to model MVA patient mutations in mice have proven difficult (Supplemental Table 1 and ref. 6), a systematic analysis of the mitotic phenotypes of the currently available models is likely to provide valuable insight into the extent to which mitosis-related abnormalities might contribute to the pathological heterogeneity. To do so, we subjected $B u b R 1^{\mathrm{H} / \mathrm{X} 753}, B u b R 1^{\mathrm{H} / \mathrm{L} 1002 \mathrm{P}}$, and $B u b R 1^{\mathrm{H} / \mathrm{H}} \mathrm{MEFs}$ and multiple tissues from $B u b R 1^{\mathrm{H} / \mathrm{L} 1002 \mathrm{P}}$ and $B u b R 1^{\mathrm{H} / \mathrm{H}}$ adult mice to the same mitotic and karyotypic tests that we conducted on mice modeling heterozygous BUBR1 MVA mutations (Figures 3 and 4).

We detected exceptionally high rates of both aneuploidy and PCS in passage-5 BubR $1^{\mathrm{H} / \mathrm{X} 753}$ MEFs (Figure $8 \mathrm{~A}$ and Supplemental Table 2), similar to earlier data on $B u b R 1^{-/ H}$ MEFs (65\% versus $72 \%$ aneuploidy, respectively) (8). This is in accordance with the fact that $B u b R 1^{\mathrm{H} / \mathrm{X} 753}$ and $B u b R 1^{-/ \mathrm{H}}$ mice both die shortly after birth and express similar BUBR1 levels in MEFs (Figure 6, A and B). Aneuploidy rates were also relatively high in $B u b R 1^{\mathrm{H} / \mathrm{L} 1002 \mathrm{P}} \mathrm{MEFs}$, reaching virtually the same levels as observed in $B u b R 1^{\mathrm{H} / \mathrm{H}} \mathrm{MEFs}$ (Figure 8A and Supplemental Table 2). The only notable difference between the 2 genotypes was the dramatic difference in PCS (Figure 8A), indicating that the presence of BUBR1 $1^{\mathrm{L} 1002 \mathrm{P}}$ protein interferes with the cell's ability to sustain strong bonds between duplicated chromosomes before anaphase onset. To examine the aneuploidy phenotypes of these biallelic MVA mutants in greater depth, we performed single-cell DNA sequencing on asynchronously growing passage-5 $B u b R 1^{+/+}, B u b R 1^{\mathrm{H} / \mathrm{L} 1002 \mathrm{P}}, B u b R 1^{\mathrm{H} / \mathrm{H}}$, and $B u b R 1^{\mathrm{H} / \mathrm{X} 753}$ MEFs. In this analysis, nearly all $B u b R 1^{\mathrm{H} / \mathrm{X} 753} \mathrm{MEFs}$ exhibited whole-chromosome aneuploidy, in contrast with nearly half of $B u b R 1^{\mathrm{H} / \mathrm{L} 1002 \mathrm{P}}$ and $B u b R 1^{\mathrm{H} / \mathrm{H}} \mathrm{MEFs}$ (Figure 8B). In all 3 mutants, most aneuploidy resulted from chromosome gains (Figure 8, C and D). BubR1 $1^{\mathrm{H} / \mathrm{X} 753}$ MEFs not only had the highest aneuploidy incidence, but also much more complex aneuploidization than $B u b R 1^{\mathrm{H} / \mathrm{L} 1002 \mathrm{P}}$ and $B u b R 1^{\mathrm{H} / \mathrm{H}} \mathrm{MEFs}$, as evidenced by the increased number of chromosomes impacted per cell (Figure 8, E and F, and Supplemental Figure 9C). Furthermore, in all 3 mutants, numerical changes occurred across a broad spectrum of chromosomes with little repetition (Supplemental Figure 9, A and B). Only BubR1 $1^{\mathrm{H} / \mathrm{X} 733} \mathrm{MEFs}$ had a few chromosomes that were more frequently gained, including chromosomes 2, 6, 8, and 10 (Supplemental Figure 9A). Chromosome losses were infrequent in $B u b R 1^{\mathrm{H} / \mathrm{H}}$ MEFs compared with $B u b R 1^{\mathrm{H} / \mathrm{X} 753}$ and $B u b R 1^{\mathrm{H} / \mathrm{L} 1002 \mathrm{P}} \mathrm{MEFs}$ (Supplemental Figure 9B). No increases in structural or segmental aneuploidy were observed in any of the biallelic MVA mutant MEFs compared to BubR $1^{+/+}$MEFs (Supplemental Figure 9, D and E). Collectively, these data indicate that early postnatal lethality, as observed in $B u b R 1^{\mathrm{H} / \mathrm{X} 753}$ mice, does seem to correlate with increases in both the rate and complexity of aneuploidization.

Consistent with the similarity in aneuploidy phenotypes, $B u b R 1^{\mathrm{H} / \mathrm{L} 1002 \mathrm{P}}$ and $B u b R 1^{\mathrm{H} / \mathrm{H}} \mathrm{MEFs}$ had the same chromosome missegregation rates, even though the prevalence of the actual types of segregation errors differed to some extent (Figure 8G). SAC activity was severely compromised in both $B u b R 1^{\mathrm{H} / \mathrm{L} 1002 \mathrm{P}}$ and $B u b R 1^{\mathrm{H} / \mathrm{H}}$ MEFs (Figure $8 \mathrm{H}$ ), as was BUBR1 accumulation at unattached kinetochores at the onset of mitosis (Supplemental Figure 10, A and B). Similar to the high increase in aneuploidy observed in $B u b R 1^{\mathrm{H} / \mathrm{X} 753} \mathrm{MEFs}$, these cells also exhibited significantly higher rates of chromosome missegregation compared with $B u b R 1^{\mathrm{H} / \mathrm{L} 1002 \mathrm{P}}$ and 
A
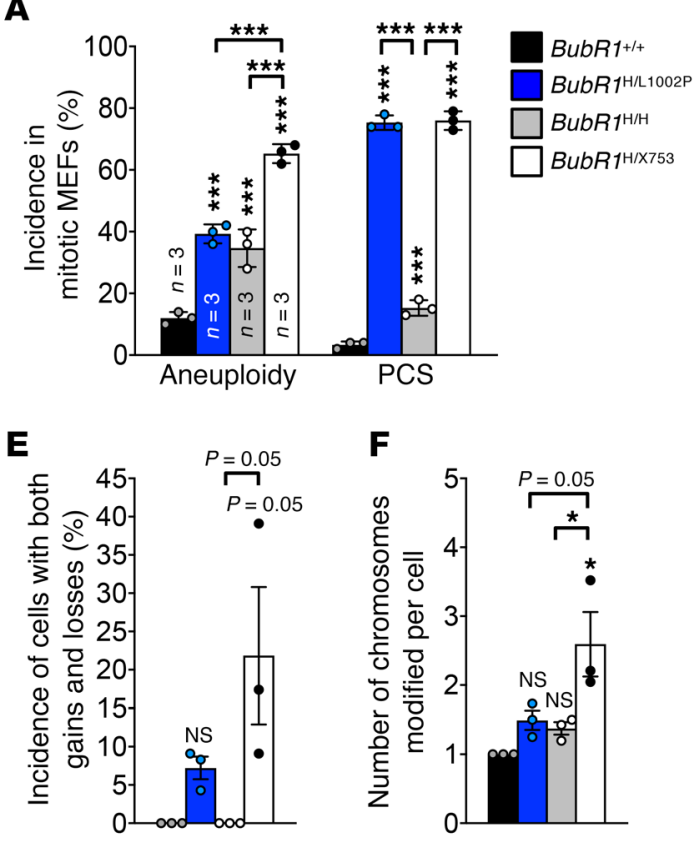
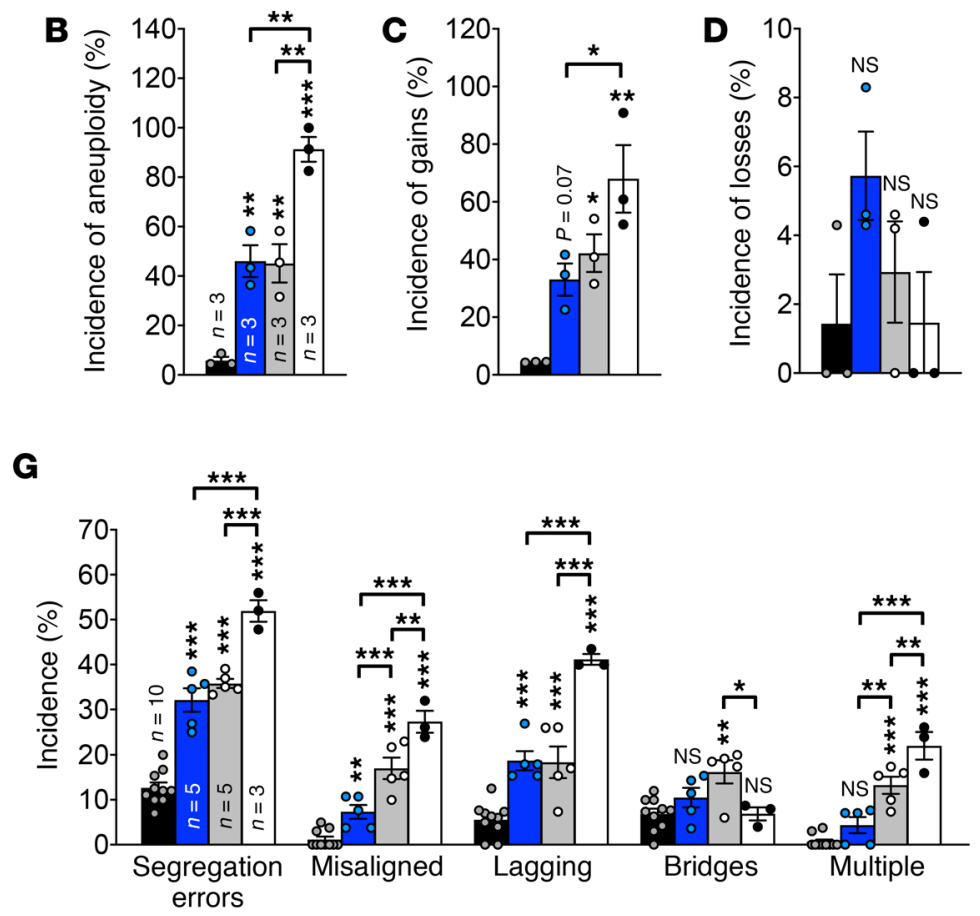

H

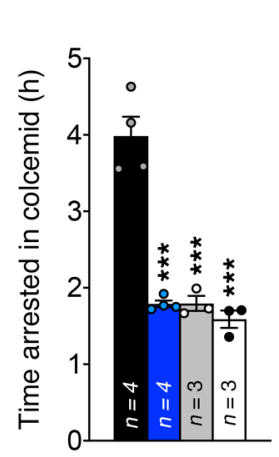

I

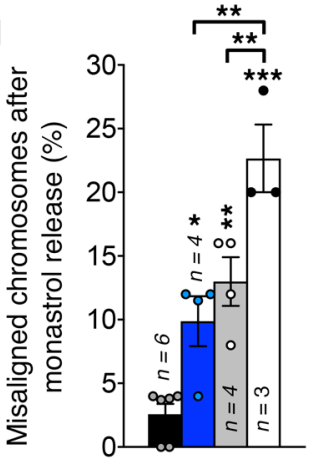

$\mathbf{J}$

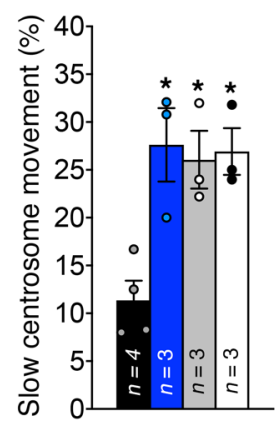

$\mathbf{K}$

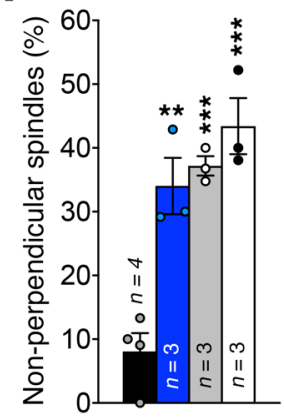

$\mathbf{L}$

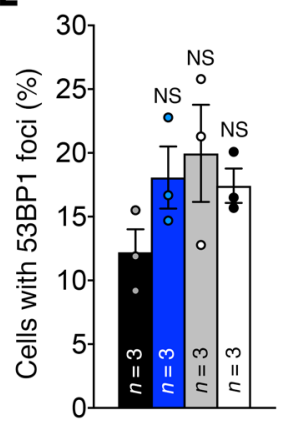

Figure 8. Phenotypically diverse MVA models have similar mitotic defects. (A) Incidence of aneuploidy and PCS in mitotically arrested passage-5 MEFs. $B u b R 7^{H / H}$ values are as previously reported (8). (B-F) Numerical aneuploidy assessments by single-cell DNA sequencing of the indicated passage- 5 MEFs. Each $n$ is as indicated in B. (C) Chromosome segregation errors in passage-5 MEFs assessed by live-cell imaging of MEFs expressing H2B-mRFP. (H) Colcemid-challenge assay on passage-5 MEFs measuring SAC activity. (I) Error correction in the indicated passage-5 MEFs assessed by monastrol washout assay. (J and K) Incidence of slow centrosome movement (J) and non-perpendicular spindles (K) in the indicated passage-5 MEFs. (L) Percentage of passage-5 MEFs with DNA damage, as determined by the presence of 53BP1 foci. Each $n$ indicates independent MEF lines. BubR $1^{+/+}$controls in $\mathbf{H}$ and $\mathbf{J}$ are as shown in Figure 3, C and E. Data are presented as mean \pm SD $(\mathbf{A})$ and mean \pm SEM (B-L), and dots represent individual samples. Statistical significance was determined using 1-way ANOVA with the Holm-Šídák post hoc test. ${ }^{*} P<0.05 ;{ }^{*} P<0.01$; ${ }^{* *} P<0.001$. NS, not significant.

$B u b R 1^{\mathrm{H} / \mathrm{H}} \mathrm{MEFs}$, although SAC activity was similarly impaired in all 3 mutants (Figure 8, G and H). However, error correction was more severely compromised in $B u b R 1^{\mathrm{H} / \mathrm{X} 753} \mathrm{MEFs}$ than in $B u b R 1^{\mathrm{H} / \mathrm{L} 1002 \mathrm{P}}$ and $B u b R 1^{\mathrm{H} / \mathrm{H}}$ MEFs (Figure 8I), providing a plausible explanation for their higher rates of chromosome misalignment and lagging. As for monoallelic MVA mutants, biallelic mutants also had impaired centrosome movement and formed non-perpendicular spindles at increased rates, although there was no distinction in the severity of these phenotypes among the 3 biallelic mutants (Figure 8, J and K). Since $B u b R 1^{\mathrm{H} / \mathrm{H}}$ MEFs also exhibited a significant increase in chromatin bridges, we assessed DNA damage by performing IF for the double-strand break (DSB) protein, 53BP1. However, we did not observe a significant increase in DSBs in any of the mutants (Figure $8 \mathrm{~L}$ ). Thus, chromosome missegregation in biallelic MVA mutants is likely driven by a combination of defects, including reduced SAC activity, defective attachment error correction, and aberrations in centrosome movement and spindle symmetry, with the higher level of aneuploidy in $B u b R 1^{\mathrm{H} / \mathrm{X} 753}$ MEFs probably due to more defective attachment error correction machinery.

Aneuploidy rates in mitotic splenocytes from 5-month-old $B u b R 1^{\mathrm{H} / \mathrm{L} 1002 \mathrm{P}}$ and $\mathrm{BubR} 1^{\mathrm{H} / \mathrm{H}}$ mice were also markedly elevated, but unlike MEFs, to different degrees (38\% and 15\%, respectively) (Figure 9A, Supplemental Table 4, and ref. 8). The same was true for rates of PCS (Figure 9A). FISH analysis for chromosomes 4 and 7 showed that rates of karyotypic abnormalities were prominently increased in a broad spectrum of tissues from both $B u b R 1^{\mathrm{H} / \mathrm{L} 1002 \mathrm{P}}$ and $B u b R 1^{\mathrm{H} / \mathrm{H}}$ mice, with minimal differences observed between the 2 genotypes (Figure 9B and Supplemental Table 3). However, 

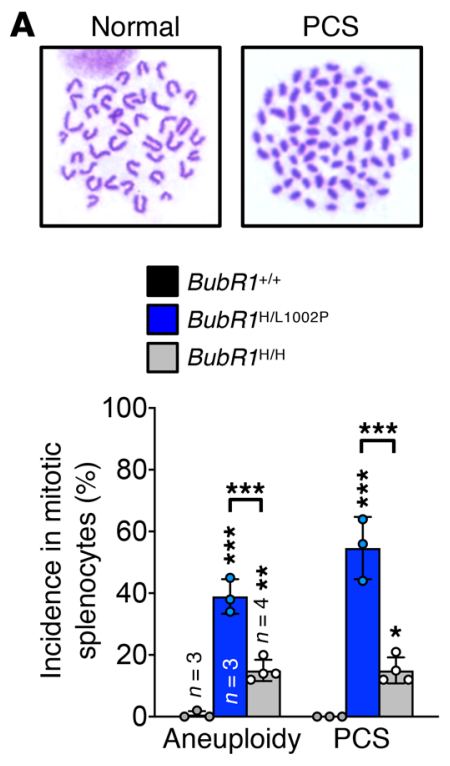

B

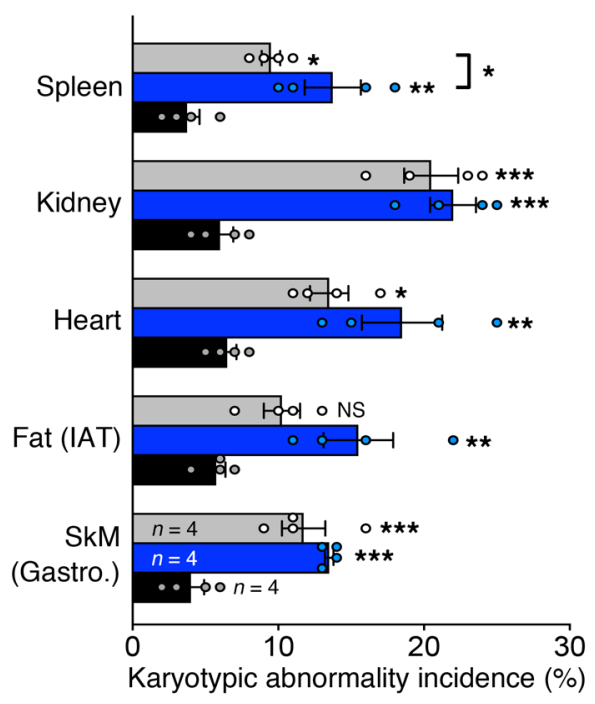

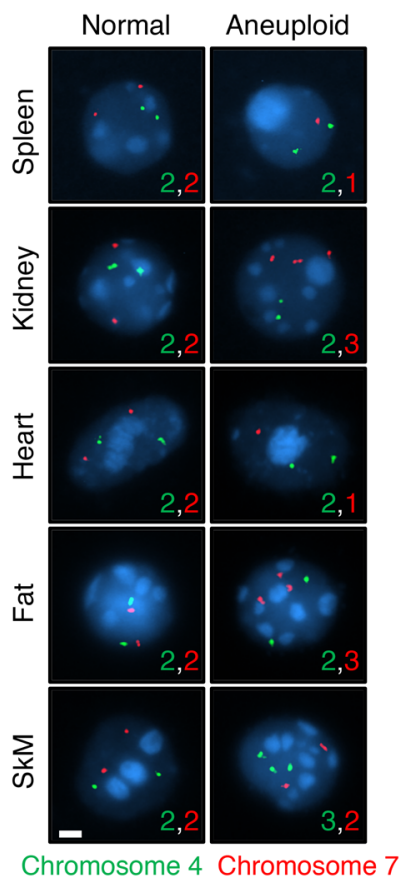

Figure 9. Progeroid MVA models exhibit severe mosaic aneuploidy. (A) Incidence of aneuploidy and PCS in mitotic splenocytes of 5-month-old mice. Images are representative of normal spreads and spreads with PCS. BubR $1^{1 / H}$ values are as previously reported (8). BubR $1^{+/+}$controls are as shown in Figure 4A. (B) Incidence of aneuploidy in tissues from 8- to 10-month-old mice assessed by FISH for chromosomes 4 and 7. Representative images shown. Scale bar: $2 \mu \mathrm{m}$. Each $n$ indicates tissues from independent mice. Data are presented as mean \pm SD $(\mathbf{A})$ and mean \pm SEM (B), and dots represent individual samples. Statistical significance was determined using 1-way ANOVA with the Holm-Šídák post hoc test. ${ }^{*} P<0.05 ;{ }^{* *} P<0.01 ;{ }^{* *} P<0.001$. NS, not significant. SkM, skeletal muscle.

aneuploidy rate or degree alone does not clearly correlate with the severity of the progeroid phenotypes observed in $B u b R 1^{\mathrm{H} / \mathrm{L} 1002 \mathrm{P}}$ and $B u b R 1^{\mathrm{H} / \mathrm{H}}$ MVA models, implying that other pathological events are key contributors or drivers.

Senescence-driven progeroid mechanisms are conserved in MVA models. Cellular senescence contributes to the development of certain progeroid phenotypes in $B u b R 1^{\mathrm{H} / \mathrm{H}}$ mice, including sarcopenia, lipodystrophy, and cataract formation $(9,10)$. Although the senescence-inducing stressors in this model remain elusive, aneuploidy alone does not seem to be sufficient to activate this cell fate program. This is largely based on the observation that several CIN models involving genes other than BubR1 are not senescence prone, despite similar or higher rates of aneuploidization than reported for the $B u b R 1^{\mathrm{H} / \mathrm{H}}$ model (27). However, 2 recent studies have suggested that aneuploidy can drive senescence, at least in vitro $(28,29)$. In one of these studies, loss of SMC1, a component of the mitotic cohesin complex, was associated with induction of both cellular senescence and PCS, but no perturbations in SMC1 were detected in senescence-prone BubR $1^{\mathrm{H} / \mathrm{H}}$ MEFs (Supplemental Figure 10C). However, in addition to the rate of aneuploidization, the possibility that karyotype complexity is an important determining factor in cell fate determination in the various CIN models cannot be excluded and requires additional studies.

To further delineate the role of senescence in MVA syndrome pathologies, we sought to determine whether senescence also drives progeria in $B u b R 1^{\mathrm{H} / \mathrm{L} 1002 \mathrm{P}}$ mice. Indeed, subcutaneous and visceral fat depots of $B u b R 1^{\mathrm{H} / \mathrm{L} 1002 \mathrm{P}}$ mice stained for senescenceassociated $\beta$-galactosidase (SA- $\beta$-gal) exhibited increased activ- ity, similar to $B u b R 1^{\mathrm{H} / \mathrm{H}}$ mice (Figure $10 \mathrm{~A}$, and data not shown). To obtain further evidence for accumulation of senescent cells in $B u b R 1^{\mathrm{H} / \mathrm{L} 1002 \mathrm{P}}$ and $B u b R 1^{\mathrm{H} / \mathrm{H}}$ fat tissue, we conducted an unbiased screen for senescence markers and components comprising the senescence-associated secretory phenotype (SASP) using a transcriptomics approach. To this end, RNA was collected from inguinal adipose tissue (IAT) from 8- to 10-month-old $B u b R 1^{\mathrm{H} / \mathrm{H}}$, $B u b R 1^{\mathrm{H} / \mathrm{L} 1002 \mathrm{P}}$, and $\mathrm{BubR} 1^{+/+}$mice, and was used for RNA sequencing. Although $B u b R 1^{\mathrm{H} / \mathrm{H}}$ and $B u b R 1^{\mathrm{H} / \mathrm{L} 1002 \mathrm{P}}$ IAT had several thousand DEGs when compared with IAT of $B u b R 1^{+/+}$mice, only 16 DEGs were observed when $B u b R 1^{\mathrm{H} / \mathrm{H}}$ and $B u b R 1^{\mathrm{H} / \mathrm{L} 1002 \mathrm{P}}$ transcriptomes were compared to each other (Figure 10B), indicating that similar biological aberrations occur in fat tissue of the 2 MVA models. Further, evaluation of the expression of the Cdkn2a locus, a well-established marker of cellular senescence and key driver of senescence in $B u b R 1^{\mathrm{H} / \mathrm{H}}$ mice $(9,10)$, revealed similarly elevated levels in both $B u b R 1^{\mathrm{H} / \mathrm{L} 1002 \mathrm{P}}$ and $B u b R 1^{\mathrm{H} / \mathrm{H}}$ fat tissue (Figure 10B). To further evaluate the senescent-cell signature in these 2 models, we interrogated our lists of upregulated DEGs for putative SASP factors by evaluating the presence of genes encoding extracellular proteins (30). One hundred ninety of the DEGs observed in the IAT between MVA models and wild-type mice encode extracellular proteins, many of which comprised known SASP functions, including proinflammatory factors, growth factors, regulators of extracellular protease activity, and other signaling factors (Figure 10C), supporting the idea that senescent cells with a bioactive secretome accumulate in fat tissue of MVA models. Next, we focused on senescence in skeletal muscle. In contrast with fat, 
A

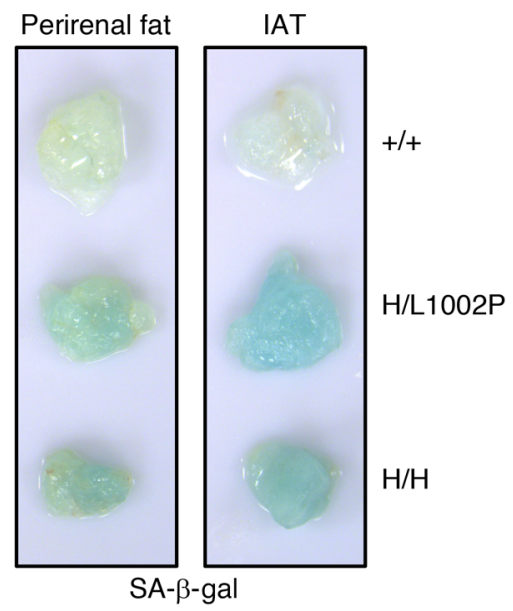

B

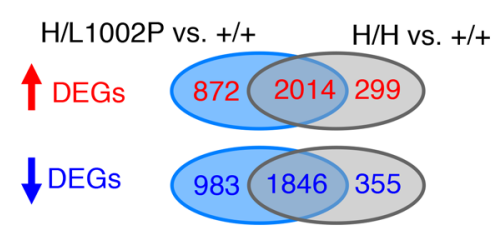

$\mathrm{H} / \mathrm{H}$ vs. H/L1002P

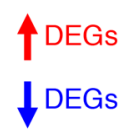

5

11 p16/p19 (Cdkn2a)

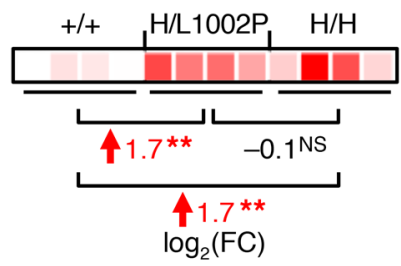

\begin{tabular}{l} 
Row Z-score \\
\hline Min
\end{tabular}

C

H/L1002P vs. +/+ H/H vs. + /+

$\uparrow$ DEGs

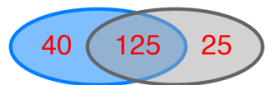

GO Extracellular Space_Putative SASP factors

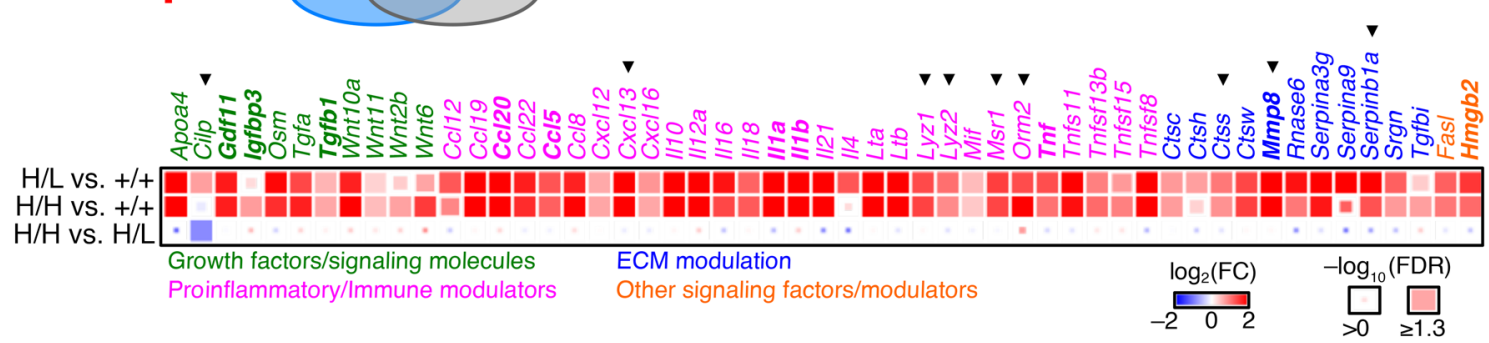

D

Skeletal muscle

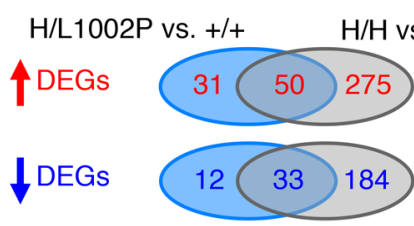

H/H vs. H/L1002P

†DEGs
$\downarrow$ DEGs

10

22
E

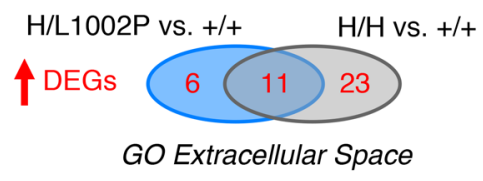

H/L vs. $+/+$ $\mathrm{H} / \mathrm{H}$ vs. $+/+$ $\mathrm{H} / \mathrm{H}$ vs. $\mathrm{H} / \mathrm{L}$

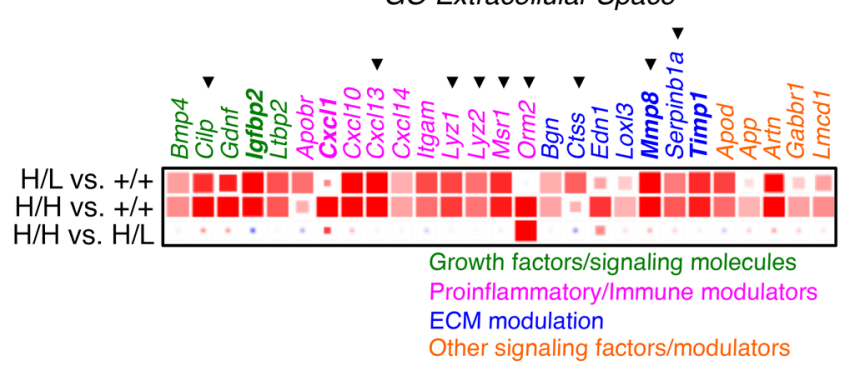

Figure 10. Senescence-mediated pathologies are conserved among MVA models. (A) Images of SA- $\beta$-gal activity in adipose tissue (perirenal and IAT) from the indicated 8- to 10-month-old mice. (B) Venn diagrams of significantly DEGs from RNA sequencing analyses of 8- to 10-month-old fat (IAT) from the indicated mice (left). $\uparrow$, upregulated genes; $\downarrow$, downregulated genes. Heatmap of expression (row Z-scores) of cell cycle inhibitors p16/p19 (Cdkn2a) in each of the individual samples (right). $n=4$ independent mice/genotype were used for analyses. FC, fold change. (C) Venn diagrams of putative SASP factors within the upregulated DEGs from $B u b R 1^{H / L 1002 P}$ versus $B u b R 1^{+/+}$and $B u b R 1^{H / H}$ versus $B u b R 1^{+/+}$RNA sequencing results from fat, determined by overlap with a gene ontology (GO) G0:0005615 "Extracellular Space" gene list (top). Heatmap of expression of 53 select putative SASP factors significantly upregulated in $B u b R 1^{H / L 1002 P}$ and/or BubR $1^{H / H}$ fat (bottom). Gene names in bold text denote established SASP factors. Arrowheads indicate putative SASP factors present in fat and skeletal muscle. (D) Venn diagrams of significantly DEGs from RNA sequencing analyses of 8- to 10-month-old gastrocnemius skeletal muscle from the indicated mice (left), as above in B. Heatmap of expression (row Z-scores) of cell cycle inhibitors p16/p19 (Cdkn2a) in each of the individual samples (right), as above in B. $n=4 B u b R 1^{+/+}$and $B u b R 1^{H / L 1002 P}$, and $n=3 B u b R 1^{H / H}$ mice were used for analyses. (E) Venn diagrams of putative

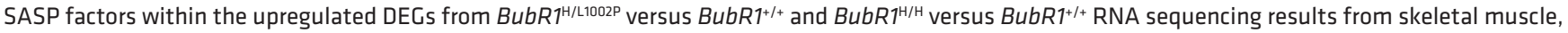
as above in C (top). Heatmap of expression of 26 select putative SASP factors significantly upregulated in $B u b R 1^{H / L 1002 P}$ and/or BubR $1^{H / H}$ skeletal muscle, as above in $\mathbf{C}$ (bottom). See Methods for statistical analyses for RNA sequencing (B and $\mathbf{D})$. ${ }^{* *} P<0.01$; ${ }^{* *} P<0.001$. NS, not significant.

$B u b R 1^{\mathrm{H} / \mathrm{H}}$ and $B u b R 1^{\mathrm{H} / \mathrm{L1} 1002 \mathrm{P}}$ gastrocnemius had only a few hundred DEGs compared with wild-type, and a higher percentage of DEGs was identified between the 2 mutants (Figure 10D). However, in accordance with increased senescence, $C d k n 2 a$ expres- sion was also significantly increased in both mutants (Figure 10D), and a substantial proportion of the upregulated DEGs also encoded putative SASP factors (Figure 10E). Although there was some overlap between putative SASP factors of $B u b R 1^{\mathrm{H} / \mathrm{L1002P}}$ and 
$B u b R 1^{\mathrm{H} / \mathrm{H}}$ muscles, $B u b R 1^{\mathrm{H} / \mathrm{H}}$ muscles contained a relatively large number of unique SASP factors, consistent with a more severe degenerative phenotype (Figure 10E). Taken together, these findings indicate that progeroid mechanisms in select $B u b R 1^{\mathrm{H} / \mathrm{H}}$ tissues are mediated by the accumulation of senescent cells, which is preserved in $B u b R 1^{\mathrm{H} / \mathrm{L} 1002 \mathrm{P}}$ mice. Furthermore, diversity in senescent cell properties (SASP composition) may underlie the difference in the severity of the progeroid skeletal muscle phenotypes between $B u b R 1^{\mathrm{H} / \mathrm{L} 1002 \mathrm{P}}$ and $B u b R 1^{\mathrm{H} / \mathrm{H}}$ mice.

\section{Discussion}

Studies of rare genetic diseases are important in that they often provide entry points for understanding cellular and organismal mechanisms that underlie more common degenerative and pathological processes, including cancer, aging, and age-related diseases (31). To learn more about the mechanisms underlying MVA syndrome and its phenotypic heterogeneity, we sought to mimic human BUBR1 MVA mutations in mice and characterize the phenotypic consequences (summarized in Supplemental Table 5). Here, our use of 4 such mutations in various combinations, with each other or in combination with a $B u b R 1^{+}$allele, unearthed several important insights into BUBR1 and its role in MVA progeria syndrome, including a better understanding of how the BUBR1 allelic effects drive pathological heterogeneity among patients.

First, our study uncovers that it is difficult to create mouse models for BUBR1 MVA syndrome patients. We show that a combination of $B U B R 1$ mutations that is compatible with postnatal viability in humans causes death during early embryogenesis in mice, most likely due to mitotic failure around the time of embryo implantation, reminiscent of mice lacking the BUBR1 binding partner BUB3 (22). A recent effort to model an MVA patient with a biallelic nonsense mutation in CEP57 supports the notion that the tolerance for MVA-associated mutations is lower in mice than in humans (6). It should be noted, however, that the BUBR1 MVA patient that our mouse model mimicked was very short-lived and died at 3.6 months of age with a broad spectrum of pathologies (2), raising the possibility that future modeling of MVA patients with longer lifespans and less severe pathologies could be more successful. On the other hand, the CEP57 patient that we recently modeled died substantially later, at 15 years of age (3), yet the corresponding mice still died as newborns (6). BubR1 $1^{-/ \mathrm{H}}$ and $B u b R 1^{\mathrm{H} / \mathrm{X} 753}$ mice also die shortly after birth. All these strains share the commonality that aneuploidy rates are relatively high. Since aneuploidy has been associated with reduced cellular fitness, impaired proteostasis, and engagement of stress response pathways (32), it is plausible that organ failure is inevitable above a certain threshold rate of mosaic aneuploidization (Supplemental Table 5).

Second, by comprehensively analyzing the $B u b R 1^{+/ L 1002 P}$ and $B u b R 1^{+/-}$mouse strains, each modeling a distinct $B U B R 1$ MVA mutation, we uncovered that carrying these heterozygous mutations is not inconsequential, but instead is associated with select MVArelated pathologies that tend to be relatively mild and typically manifest themselves only in the second year of life. The BubR $1^{+/ \mathrm{L} 1002 \mathrm{P}}$ and $B u b R 1^{+/-}$strains both exhibit a reduction in median lifespan, presumably due to reduced tumor latency. The mitotic index of lymphomas of both these mutants is significantly increased (Figure 2E) compared with lymphomas of wild-type control mice, suggesting that increased tumor cell proliferation contributes to the observed reduction in tumor latency. $B u b R 1^{+/ L 1002 \mathrm{P}}$ and $B u b R 1^{+/-}$mice also exhibit an increase in lung tumor size when challenged with DMBA, reminiscent of that previously reported for $B u b R 1^{+/ X 753}$ mice (21), but whether this is due to accelerated tumor initiation or proliferation, or both, remains to be established. $B u b R 1^{+/ X 753}$ mice, but not $B u b R 1^{+/ L 1002 P}$ and $B u b R 1^{+/-}$mice, develop multiple progeroid phenotypes observed in $B u b R 1^{\mathrm{H} / \mathrm{H}}$ mice, which establish themselves in the second year of life, including sarcopenia, lipodystrophy, and cataract formation (21). In probing the mechanism associated with these phenotypes, we found that skeletal muscle from $B u b R 1^{+/ X 753}$ mice is characterized by hyperactive mTORC1 signaling. Chronic hyperactivation of $\mathrm{mTORC} 1$ has been linked to skeletal muscle atrophy and induction of cellular senescence $(33,34)$. This, combined with studies demonstrating that inhibition of mTOR with rapamycin slows age-related deterioration in mice (26), suggests that hyperactive mTORC1 signaling may be a driver of accelerated sarcopenia in $B u b R 1^{+/ X 753}$ mice. BUBR1 and MAD2 have recently been implicated in the control of insulin signaling and metabolic homeostasis, raising the interesting possibility that disruption of this function is linked to uncontrolled mTORC1 activity (20). Collectively, these data reveal that a key feature of MVA syndrome patients, phenotypic heterogeneity, is conserved among mouse models for heterozygous BUBR1 MVA allele carriers (Supplemental Table 5).

Third, although we failed to model the MVA patient $B U B R 1^{\mathrm{L} 1012 \mathrm{P} / \mathrm{X} 753}$, we successfully created a viable MVA syndrome model using BUBR1 mutations found in MVA patients. Previously, modeling was restricted to the use of hypomorphic BubR1 alleles, mimicking a $B U B R 1$ variant found in Asian MVA patients that have no discernable mutations, but nevertheless yield low amounts of wild-type BUBR1 protein (1). However, only 1 BUBR1 MVA patient with 2 hypomorphic alleles has been reported to date (35). Although this has raised questions about the faithfulness of the model, its usefulness became more accepted with the discovery that the critical contribution of many missense mutations found in MVA syndrome patients is destabilization of the BUBR1 protein and lowering overall protein amounts (7). On the other hand, an independently generated hypomorphic model, referred to as $B u b R 1^{\mathrm{L} / \mathrm{L}}$, did not show any of the overtly detectable progeroid features of $B u b R 1^{\mathrm{H} / \mathrm{H}}$ mice, such as kyphosis, lipodystrophy, and cataract formation (36), suggesting that subtle allelic effects may have dramatic phenotypic consequences. This idea is underscored here by the observation that $B u b R 1^{\mathrm{H} / \mathrm{H}}$ and $B u b R 1^{\mathrm{H} / \mathrm{L} 1002 \mathrm{P}}$ mice have quite obvious phenotypic differences despite an inability to detect differences in overall BUBR1 protein levels in a broad spectrum of tissues. The mere difference between total BUBR1 protein levels in $B u b R 1^{\mathrm{H} / \mathrm{H}}$ and $B u b R 1^{\mathrm{H} / \mathrm{L} 1002 \mathrm{P}}$ mice is that in the latter strain the total pool consists of a mixture of BUBR1 $1^{\mathrm{WT}}$ and BUBR1 $1^{\mathrm{L} 1002 \mathrm{P}}$ protein rather than BUBR1 ${ }^{\mathrm{WT}}$ alone. Although several phenotypes of $B u b R 1^{\mathrm{H} / \mathrm{L} 1002 \mathrm{P}}$ are similar in severity to those of $B u b R 1^{\mathrm{H} / \mathrm{H}}$ mice, including lipodystrophy, cataractogenesis, and carcinogeninduced tumor susceptibility, others are definitely milder, including growth retardation, cardiac stress sensitivity, lifespan shortening, and muscle wasting. These findings are important in that they reveal that seemingly subtle allelic effects can be the cause of rather remarkable phenotypic heterogeneity, a key hallmark of MVA syndrome (Supplemental Table 5). 
Fourth, although our study does not fully resolve key longstanding questions as to how changes in BUBR1 drive a broad spectrum of highly complex pathologies, it does provide several important mechanistic insights. For instance, although BUBR1 levels do not closely track with phenotypic severity, this study identifies several critical BUBR1 thresholds in vivo. A reduction of approximately $40 \%-90 \%$ in BUBR1 protein levels results in tumor predisposition or mild premature-aging phenotypes, whereas a reduction of greater than $90 \%$ results in progeroid phenotypes or early postnatal lethality (Supplemental Table 5). The BubR1 $1^{\mathrm{X} 75 / \mathrm{L1} 1002 \mathrm{P}}$ model does not fit this scheme, however, and suggests that there are other factors driving early embryonic lethality in this case, such as extensive aneuploidization. Since aneuploidy rates also increase as these critical BUBR1 thresholds are breached, it is difficult to rule this out as a contributing factor. However, it is unlikely that differences in aneuploidization represent a major source of phenotypic heterogeneity between the $B u b R 1^{\mathrm{H} / \mathrm{H}}$ and $B u b R 1^{\mathrm{H} / L 1002 \mathrm{P}}$ models given that their mitotic phenotypes show remarkable similarity with regard to levels of SAC, error correction, and spindle geometry impairment, rates of chromosome missegregation, and aneuploidization in MEFs. Moreover, although aneuploidy rates in mitotically active splenocytes are substantially higher in the BubR $1^{\mathrm{H} / \mathrm{L1002P}}$ model, FISH-based assessments point to similar amounts of chromosome mosaicism in a wide spectrum of tissues of both $B u b R 1^{\mathrm{H} / \mathrm{H}}$ and $B u b R 1^{\mathrm{H} / \mathrm{L} 1002 \mathrm{P}}$ mice. Importantly, a recent study illustrated that chromosome segregation fidelity outside the normal tissue architecture is severely compromised, and that in vitro culture of cells on monolayers promotes aneuploidization (37). In light of these findings, aneuploidy data from cultured MEFs are unlikely to reflect in vivo aneuploidy rates. However, our method for assessment of aneuploidy in splenocytes is not subject to this caveat, as it does not involve any cells engaging in chromosome segregation while in culture. Since this method focuses only on a subset of splenocytes, namely those that are mitotically active, rates obtained are unlikely to faithfully reflect aneuploidy rates in the spleen at large. Therefore, the rates of aneuploidy obtained by FISH probably represent the most reliable relative measure for aneuploidy between the 2 MVA models. Moreover, FISH has been shown to be consistent with assessments of aneuploidy by whole-genome sequencing, underscoring its validity as a measure of aneuploidy (38). Another indication that aneuploidy alone is not a prominent source for phenotypic heterogeneity is that the progeroid phenotypes associated with BubR1 mutations are typically not observed in the now large collection of mouse strains with mutations in other key mitotic regulators $(27,39,40)$. This even includes mutants involving prominent binding partners or closely related BUBR1 family members, such as BUB3, BUB1, MAD2, and CDC2O.

Cellular senescence is one mechanism that has been causally linked to the induction of a subset of progeroid phenotypes in $B u b R 1^{\mathrm{H} / \mathrm{H}}$ mice, including sarcopenia, cataracts, and lipodystrophy $(8,10)$, but how BUBR1 aberration induces cellular senescence remains to be determined. However, our side-by-side comparison of the $B u b R 1^{\mathrm{H} / \mathrm{H}}$ and $B u b R 1^{\mathrm{H} / \mathrm{L} 1002 \mathrm{P}}$ models provides important information regarding the relationship between BUBR1 and cellular senescence. First, we demonstrate that there is a strong conservation of senescence-dependent phenotypes between the 2 independent models. Over recent years, it has become increas- ingly clear that the bioactive secretome of senescent cells is an important driver of tissue degeneration, but a comprehensive assessment of the SASP in the context of MVA syndrome has not been conducted. Here, using an RNA sequencing approach, we identified a broad spectrum of putative SASP factors implicated in senescent cell-dependent degeneration in fat and skeletal muscle. These findings suggest that there is substantially more heterogeneity in bioactive secretomes in vivo than expected based on initial in vitro data (41). Further, we uncovered that the number of putative SASP factors is different in fat and skeletal muscle irrespective of the MVA syndrome-causing BubR1 alleles, with fat from both $B u b R 1^{\mathrm{H} / \mathrm{H}}$ and $B u b R 1^{\mathrm{H} / L 1002 \mathrm{P}}$ mice showing a rather large number of factors compared with muscle. Importantly, rates of senescence are similar between $B u b R 1^{\mathrm{H} / \mathrm{H}}$ and $B u b R 1^{\mathrm{H} / \mathrm{L} 1002 \mathrm{P}}$ mice based on levels of Cdkn2a expression. This holds true for both fat and skeletal muscle. For fat this is perhaps not surprising because lipodystrophy severity is similar between $B u b R 1^{\mathrm{H} / \mathrm{H}}$ and $B u b R 1^{\mathrm{H} / \mathrm{L1} 002 \mathrm{P}}$ mice, and their SASP factors are highly conserved. In the case of skeletal muscle, however, $B u b R 1^{\mathrm{H} / \mathrm{H}}$ mice exhibit more severe tissue wasting than $B u b R 1^{\mathrm{H} / L 1002 \mathrm{P}}$ mice. Because $B u b R 1^{\mathrm{H} / \mathrm{H}}$ muscles seem to have a more complex secretome than their $B u b R 1^{\mathrm{H} / L 1002 \mathrm{P}}$ counterparts, one plausible explanation would be that senescent cells that accumulate in $B u b R 1^{\mathrm{H} / \mathrm{H}}$ muscles have more profound degenerative properties. This may be due to differences in senescence-inducing stressors in these mice due to allelic effects, or differential evolution of cells once they enter the senescent state, as previously suggested (42). However, the possibility that degenerative mechanisms beyond senescence may also contribute to the muscle pathology in $B u b R 1^{\mathrm{H} / \mathrm{H}}$ mice cannot be excluded.

In conclusion, the use of mice mimicking BUBR1 MVA mutations has unearthed many insights regarding the physiological relevance of BUBR1, and its role in MVA progeria syndrome. The results of our study predict that future modeling of other MVA mutations is likely to uncover important new clues about BUBR1-mediated biological processes in cancer, aging, and age-related diseases. Additionally, it will be imperative to establish mutant mouse models designed to identify the senescence-inducing stressors activated by BUBR1 dysfunction. Likewise, it will be important to establish mouse models that further probe the in vivo relevance of the recently discovered non-mitotic BUBR1 functions in receptor signaling, and control of CDC2O-activated APC/C substrate stability $(20,43,44)$.

\section{Methods}

Mouse strains. BubR1 $1^{+/ \mathrm{L} 1002 \mathrm{P}}$ mice were generated by converting the first thymidine of BubR1 codon 1002 into a cytosine (CTT>CCT) using previously described gene targeting in mouse embryonic stem (ES) cells (21). See the Supplemental Figure 1 legend for details. The

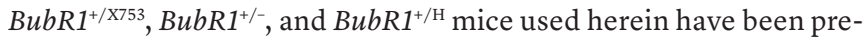
viously described $(8,21)$. All mice used in this study were on a mixed $129 / \mathrm{Sv} \times \mathrm{C} 57 \mathrm{BL} / 6$ background, and were housed in a pathogenfree barrier facility.

$M E F$ generation and culture. MEFs were generated as previously described (22). Primary MEFs were cultured and frozen at passages 2 and 3 , and vials thawed and used for experiments between passages 4 and 6. At least 3 independent MEF lines were used for each experiment, unless otherwise noted. 
Western blotting and quantifications. Western blot analyses were performed as previously described (8). MEF and tissue lysates were prepared as previously described (14). For assessments of BUBR1 stability, passage-5 MEFs were treated with cycloheximide, MG132, or the combination for 5 hours, as previously described (7). The mouse anti-BUBR1 (BD Transduction, catalog 612503), rabbit anti- $\alpha$-tubulin (Cell Signaling Technology, catalog 2125), rabbit anti-phospho-p70 S6 kinase (Thr389; Cell Signaling Technology, catalog 9208), and rabbit anti-phospho-4EBP1 (Thr37/46; Cell Signaling Technology, catalog 2855) primary antibodies were used at a dilution of 1:1,000. PonS staining $(1.1 \mathrm{~g} / \mathrm{mL}$ Ponceau S (Sigma-Aldrich) in $1 \%$ glacial acetic acid) served as a loading control unless otherwise noted. BUBR1 levels were quantified using Image software (NIH) and were normalized to levels of $\alpha$-tubulin or total protein using PonS.

Quantitative reverse transcriptase PCR. Total RNA was extracted from passage- 5 MEFs using the RNeasy mini kit (Qiagen, catalog 74104). cDNA synthesis and qRT-PCR were performed as previously described (45). See the Supplemental Figure 2 legend for details.

Karyotyping and interphase FISH. Chromosome counts of metaphase spreads from MEFs and mouse splenocytes were performed at passage- 5 and 5 months of age, respectively, as previously described (22). Fifty spreads were analyzed per sample. Interphase FISH using probes for mouse chromosomes $4 \mathrm{Eq} 2$ and $7 \mathrm{qA} 1$ were performed on single-cell suspensions generated from MEFs and various mouse tissues as previously described (14). One hundred interphase cells were analyzed per sample. Karyotype analysis by single-cell DNA sequencing was performed on passage- $5 \mathrm{MEFs}$ as previously described $(46,47)$. At least 3 independent MEF lines were sequenced per genotype, and 19-24 cells were sequenced per line. Whole chromosomal and structural aneuploidies were determined using AneuFinder software (46).

IF and mitotic assays. Indirect IF was performed and quantified using Image J software as previously described (45). Assessments of BUBR1 (BD Transduction, catalog 612503) and SMC1 (Abcam, catalog ab133643) centromere localization (Antibodies, Inc., catalog 15-234001) (15); centrosome movement (anti- $\gamma$-and $\alpha$-tubulin; Sigma-Aldrich, catalog T5192 and T9026) and spindle geometry (anti- $\gamma$-tubulin and -phospho-histone H3 [Ser10, pHH3]; Sigma-Aldrich, catalog T6557 and EMD Millipore, catalog 06-570) (45); and DNA damage (anti-53BP1, Novus Biologicals, catalog NB100-305) (48) were performed as previously described. A laser-scanning microscope (LSM 880; Carl Zeiss) with an inverted microscope (Axiovert 100M; Carl Zeiss) was used for imaging. The monastrol washout assay was performed as previously described (15). Briefly, $100 \mu \mathrm{M}$ monastrol (Enzo Life Sciences, catalog BML-GR322) was added to passage-5 MEFs for 60 minutes, followed by the addition of $10 \mu \mathrm{M}$ MG132 (Sigma-Aldrich, catalog C2211) plus monastrol for an additional 60 minutes. Cells were then released in 10 $\mu \mathrm{M}$ MG132 alone for 90 minutes and fixed in $4 \%$ paraformaldehyde for 10 minutes and stained with Hoechst. Metaphase cells with 1 or more misaligned chromosomes were scored as containing uncorrected misalignments. At least 3 independent MEF lines per genotype were used for analyses, and at least 25 metaphase cells were analyzed per line.

Live-cell imaging analyses. Chromosome segregation analyses and colcemid challenge assays were performed on passage 5-6 primary MEFs stably expressing H2B-mRFP, as previously described (15). Colcemid was added at $0.5 \mu \mathrm{g} / \mathrm{mL}$ (Karyomax, Gibco, catalog 15210040). At least 23 and 10 cells per line were analyzed for chromosome segregation and colcemid challenge assays, respectively.
Analysis of age-related phenotypes. Mouse cohorts for lifespan and healthspan studies were generated, and animals were evaluated biweekly for cataracts and kyphosis as previously described (9). For evaluation of survival and cancer deaths, moribund mice were euthanized and all major organs were screened grossly for overt tumors, as previously described (9). Exercise ability by treadmill testing was performed and work output was calculated as previously described (10). Forelimb grip strength testing was performed as previously described (49). Body composition and total body fat were determined using an EchoMRI-100 QNMR instrument (Echo Medical Systems), as previously described (49). Total body lean and fat mass were recorded, and total body fat percentages were determined by dividing fat mass by body mass. Bone mineral density (BMD) was determined with dualenergy x-ray absorptiometry scanning (Lunar PIXImus densitometer), as previously described (49). Cardiac stress tolerance was assessed using a chronic isoproterenol test, similarly to previously described studies (13). Terminal healthspan analyses of fat depot weights and collection of fat and skeletal muscle were performed as previously described $(8,9)$. Fat depot weights were normalized to the length of the right femur. Skeletal muscle (gastrocnemius) and fat (IAT) were fixed in $10 \%$ normal buffered formalin, embedded in paraffin, and $5-\mu \mathrm{m}$ sections were stained with hematoxylin and eosin (H\&E) as previously described (9). Skeletal muscle (gastrocnemius) fiber and fat (IAT) adipocyte sizes were determined as previously described (9), with the following modifications: cross-sectional area measurements of 150 fibers or adipocytes were made using ImageJ software. The mean of these measurements was then obtained per sample, and individual means were then averaged per group.

Tumor analyses. For spontaneous tumor analyses, mice were maintained until 18-20 months of age, and were then euthanized and grossly examined for overt tumors in all major organs, as described above. Carcinogen-induced tumorigenesis studies were performed with administration of DMBA as previously described (45). We note that studies on $B u b R 1^{+/ L 1002 P}$ and $B u b R 1^{+/-}$cohorts were conducted several years apart and with different batches of DMBA, and that variation between experiments can be observed ( $20 \%-55 \%$ lung tumor incidence, and $\sim 0.65-2.2$ average tumor numbers in wild-type mice). Animals were euthanized at 4 months of age, and screened for the presence of overt tumors, including skin and lung. All tumors were counted, and the longest diameter measured at the time of collection or from images taken at time of collection. Lung tumor volumes were calculated using diameter measurements as previously described (21). To assess the mitotic index of spontaneous lymphatic tumors, mitotic cells were visualized by IF labeling of 2 paraffin sections separated by $150 \mu \mathrm{m}$ for $\mathrm{pHH} 3$ (EMD Millipore, catalog 06-570) as previously described $(6,47)$.

$S A-\beta$-gal staining. Whole-mount SA- $\beta$-gal staining on fat (IAT) was performed as previously described (13). At least 3 animals were analyzed.

RNA library preparation, sequencing, and bioinformatic analyses. RNA was extracted from fat tissue or gastrocnemius muscle from 8to 10-month-old mice using the RNeasy lipid and fibrous tissue kits, respectively (Qiagen, catalog 74704 and 1023539), and from gastrocnemius muscles from 3-month-old mice using the RNeasy fibrous tissue kit: $n=4 B u b R 1^{+/+}, B u b R 1^{\mathrm{H} / \mathrm{L} 1002 \mathrm{P}}$, and $B u b R 1^{\mathrm{H} / \mathrm{H}}$ mice for IAT; $n=4 B u b R 1^{+/+}$and $B u b R 1^{\mathrm{H} / \mathrm{L} 1002 \mathrm{P}}$ mice and $n=3$ for $B u b R 1^{\mathrm{H} / \mathrm{H}}$ mice for 8- to 10-month-old gastrocnemius; and $n=3 B u b R 1^{+/+}, B u b R 1^{+/ L 1002 P}$, and $B u b R 1^{+/ X 753}$ mice for 3-month-old gastrocnemius. Library preparation and RNA sequencing were performed as previously described 
(47). Fastq files of paired-end reads were aligned with Tophat 2.0.14 to the UCSC reference genome mm10 using Bowtie2 2.2.6 with default parameters. Differential expression analyses were performed using $\mathrm{R}$ package DESeq2 1.10.1 after removing genes with average raw counts less than 10 (47). Genes with lfcMLE (unshrunken maximum likelihood estimate of $\log _{2}$ fold change produced by DESeq2) greater than 0.45 or less than -0.45 , and FDR less than 0.05 were considered significantly differentially expressed. Functional enrichment analyses for DEGs from the 3-month-old monoallelic skeletal muscle samples was performed using the STRING database, version 11 (https:// string-db.org; ref. 25). Biological processes with FDR less than 0.05 were considered significantly enriched, and were classified into functional groups. For 8- to 10-month-old biallelic skeletal muscle and fat samples, putative SASP factor genes were extracted from the Gene Ontology Consortium (Mus musculus MGI), and QuickGO database for the annotation GO:0005615 "Extracellular Space" (30, 50, 51). Gene lists from both reference databases were merged, resulting in the identification of 1845 factors. SASP gene lists were then compared to differentially upregulated gene lists. Heatmaps were generated with Morpheus (Broad Institute; https://software.broadinstitute.org/ morpheus). For p16/p19 (Cdkn2a) expression heatmaps row $Z$-scores were used, and for putative SASP factor and mTORC1-related gene expression heatmaps lfcMLE values were used.

Statistics. Statistical significance was determined using the logrank test, 2-tailed Fisher's exact test, 1-way ANOVA with a HolmŚídák post hoc test, 2-tailed unpaired $t$ test, and Mann-Whitney $U$ test as noted in the figure legends. For 1-way ANOVA with Holm-Šídák correction for multiple comparisons, all groups were compared, with the exception of Figure 2E; Figure 3, A-D; Supplemental Figure 2A; and Supplemental Figure 5 where groups were compared to wild-type controls only, and where select comparisons were made (Supplemental Figure 2, C and E) as noted in the legends. All tests were performed using GraphPad Prism software, except for the 2-tailed unpaired $t$ test, which was performed using Microsoft Excel. A P value less than 0.05 was considered statistically significant. Sample sizes for all animal studies were chosen based on previously published studies in which differences were observed. The experiments were not randomized, and the investigators were not blinded.

Study approval. All animal procedures were reviewed and approved by the Institutional Animal Care and Use Committee (IACUC) at the Mayo Clinic, Rochester, Minnesota (protocol A00002189).

Data availability. RNA sequencing and single-cell DNA sequencing data have been deposited into the National Center for Biotechnology Information's Gene Expression Omnibus under the accession numbers GSE134781 (3-month-old monoallelic skeletal muscle data) and GSE134780 (8- to 10-month-old biallelic skeletal muscle and fat data) and into the European Nucleotide Archive under the accession number PRJEB33810.

\section{Author contributions}

JMVD, CJS, HL, CZ, and DJB designed the research studies. CJS, KBJ, GGN, DJB, IS, CZ, and WHVD performed experiments. FF and $\mathrm{BB}$ performed karyotyping via single-cell whole-genome sequencing. All authors contributed to the analysis and interpretation of data. JMVD and CJS wrote the manuscript, and all authors edited the manuscript. JMVD directed and supervised all aspects of the study.

\section{Acknowledgments}

We thank Liviu Malureanu for initiating the project and the members of the van Deursen lab for helpful discussions, feedback, or help with methods. We thank the Cytogenetics and Sequencing Cores of the Medical Genome Facility, Mayo Clinic, for sample processing and technical support. This work was supported by grants from the NIH (R01 CA096985) and the Glenn Foundation for Medical Research to JMVD.

Address correspondence to: Jan M. van Deursen, Mayo Clinic, 200 First St. SW, Rochester, Minnesota 55905, USA. Phone: 507.284.2524; Email: vandeursen.jan@mayo.edu.
1. Matsuura S, et al. Monoallelic BUB1B mutations and defective mitotic-spindle checkpoint in seven families with premature chromatid separation (PCS) syndrome. Am JMed Genet A. 2006;140(4):358-367.

2. Hanks S, et al. Constitutional aneuploidy and cancer predisposition caused by biallelic mutations in BUB1B. Nat Genet. 2004;36(11):1159-1161.

3. Snape K, et al. Mutations in CEP57 cause mosaic variegated aneuploidy syndrome. Nat Genet. 2011;43(6):527-529.

4. Yost S, et al. Biallelic TRIP13 mutations predispose to Wilms tumor and chromosome missegregation. Nat Genet. 2017;49(7):1148-1151.

5. García-Castillo H, Vásquez-Velásquez AI, Rivera $\mathrm{H}$, Barros-Núñez P. Clinical and genetic heterogeneity in patients with mosaic variegated aneuploidy: delineation of clinical subtypes. Am JMed Genet A. 2008;146A(13):1687-1695.

6. Aziz K, et al. Mosaic-variegated aneuploidy syndrome mutation or haploinsufficiency in Cep57 impairs tumor suppression. J Clin Invest. 2018;128(8):3517-3534.

7. Suijkerbuijk SJ, van Osch MH, Bos FL, Hanks S,
Rahman N, Kops GJ. Molecular causes for BUBR1 dysfunction in the human cancer predisposition syndrome mosaic variegated aneuploidy. Cancer Res. 2010;70(12):4891-4900.

8. Baker DJ, et al. BubR1 insufficiency causes early onset of aging-associated phenotypes and infertility in mice. Nat Genet. 2004;36(7):744-749.

9. Baker DJ, et al. Opposing roles for p16Ink4a and p19Arf in senescence and ageing caused by BubR1 insufficiency. Nat Cell Biol. 2008;10(7):825-836.

10. Baker DJ, et al. Clearance of p16Ink4a-positive senescent cells delays ageing-associated disorders. Nature. 2011;479(7372):232-236.

11. Baker DJ, Weaver RL, van Deursen JM. p21 both attenuates and drives senescence and aging in BubR1 progeroid mice. Cell Rep. 2013;3(4):1164-1174.

12. Baker DJ, Jeganathan KB, Malureanu L, PerezTerzic C, Terzic A, van Deursen JM. Early agingassociated phenotypes in Bub3/Rae1 haploinsufficient mice. J Cell Biol. 2006;172(4):529-540.

13. Baker DJ, et al. Naturally occurring p16(Ink4a)positive cells shorten healthy lifespan. Nature.
2016;530(7589):184-189.

14. Baker DJ, et al. Increased expression of BubR1 protects against aneuploidy and cancer and extends healthy lifespan. Nat Cell Biol. 2013;15(1):96-102.

15. Weaver RL, Limzerwala JF, Naylor RM, Jeganathan KB, Baker DJ, van Deursen JM. BubR1 alterations that reinforce mitotic surveillance act against aneuploidy and cancer. Elife. 2016;5:e16620.

16. Musacchio A. The molecular biology of spindle assembly checkpoint signaling dynamics. Curr Biol. 2015;25(20):R1002-R1018.

17. Holland AJ, Cleveland DW. Boveri revisited: chromosomal instability, aneuploidy and tumorigenesis. Nat Rev Mol Cell Biol. 2009;10(7):478-487.

18. Ruchaud S, Carmena M, Earnshaw WC. Chromosomal passengers: conducting cell division. Nat Rev Mol Cell Biol. 2007;8(10):798-812.

19. Suijkerbuijk SJ, Vleugel M, Teixeira A, Kops GJ. Integration of kinase and phosphatase activities by BUBR1 ensures formation of stable kinetochore-microtubule attachments. Dev Cell. 2012;23(4):745-755. 
20. Choi E, Zhang X, Xing C, Yu H. Mitotic checkpoint regulators control insulin signaling and metabolic homeostasis. Cell. 2016;166(3):567-581.

21. Wijshake T, Malureanu LA, Baker DJ, Jeganathan KB, van de Sluis B, van Deursen JM. Reduced life- and healthspan in mice carrying a mono-allelic BubR1 MVA mutation. PLoS Genet. 2012;8(12):e1003138.

22. Babu JR, Jeganathan KB, Baker DJ, Wu X, Kang-Decker N, van Deursen JM. Rae1 is an essential mitotic checkpoint regulator that cooperates with Bub3 to prevent chromosome missegregation. JCell Biol. 2003;160(3):341-353.

23. van Ree JH, Nam HJ, Jeganathan KB, Kanakkanthara A, van Deursen JM. Pten regulates spindle pole movement through Dlg1-mediated recruitment of Eg5 to centrosomes. Nat Cell Biol. 2016;18(7):814-821.

24. Naylor RM, Jeganathan KB, Cao X, van Deursen JM. Nuclear pore protein NUP88 activates anaphase-promoting complex to promote aneuploidy. J Clin Invest. 2016;126(2):543-559.

25. Szklarczyk D, et al. STRING v11: protein-protein association networks with increased coverage, supporting functional discovery in genomewide experimental datasets. Nucleic Acids Res. 2019;47(D1):D607-D613.

26. Harrison DE, et al. Rapamycin fed late in life extends lifespan in genetically heterogeneous mice. Nature. 2009;460(7253):392-395.

27. Naylor RM, van Deursen JM. Aneuploidy in cancer and aging. Annu Rev Genet. 2016;50:45-66.

28. Andriani GA, et al. Whole Chromosome Instability induces senescence and promotes SASP. Sci Rep. 2016;6:35218.

29. Macedo JC, et al. FoxM1 repression during human aging leads to mitotic decline and aneuploidy-driven full senescence. Nat Commun.
2018;9(1):2834.

30. Ashburner M, et al. Gene ontology: tool for the unification of biology. The Gene Ontology Consortium. Nat Genet. 2000;25(1):25-29.

31. Gordon LB, Rothman FG, López-Otín C, Misteli T. Progeria: a paradigm for translational medicine. Cell. 2014;156(3):400-407.

32. Sheltzer JM, Amon A. The aneuploidy paradox: costs and benefits of an incorrect karyotype. Trends Genet. 2011;27(11):446-453.

33. Castets $P$, et al. Sustained activation of mTORC1 in skeletal muscle inhibits constitutive and starvation-induced autophagy and causes a severe, late-onset myopathy. Cell Metab. 2013;17(5):731-744.

34. Laberge RM, et al. MTOR regulates the pro-tumorigenic senescence-associated secretory phenotype by promoting IL1A translation. Nat Cell Biol. 2015;17(8):1049-1061.

35. Ochiai H, et al. TALEN-mediated single-basepair editing identification of an intergenic mutation upstream of BUB1B as causative of PCS (MVA) syndrome. Proc Natl Acad Sci US A. 2014;111(4):1461-1466.

36. Kyuragi R, et al. BubR1 insufficiency inhibits neointimal hyperplasia through impaired vascular smooth muscle cell proliferation in mice. Arterioscler Thromb Vasc Biol. 2015;35(2):341-347.

37. Knouse KA, Lopez KE, Bachofner M, Amon A. Chromosome segregation fidelity in epithelia requires tissue architecture. Cell. 2018;175(1):200-211.e13.

38. Bakker B, et al. Single-cell sequencing reveals karyotype heterogeneity in murine and human malignancies. Genome Biol. 2016;17(1):115.

39. Santaguida S, Amon A. Short- and long-term effects of chromosome mis-segregation and aneuploidy. Nat Rev Mol Cell Biol. 2015;16(8):473-485.
40. Holland AJ, Cleveland DW. Losing balance: the origin and impact of aneuploidy in cancer. $E M B O$ Rep. 2012;13(6):501-514.

41. Coppé JP, et al. Senescence-associated secretory phenotypes reveal cell-nonautonomous functions of oncogenic RAS and the p53 tumor suppressor. PLoS Biol. 2008;6(12):2853-2868.

42. van Deursen JM. The role of senescent cells in ageing. Nature. 2014;509(7501):439-446.

43. Yang Y, et al. A Cdc20-APC ubiquitin signaling pathway regulates presynaptic differentiation. Science. 2009;326(5952):575-578.

44. Kim AH, et al. A centrosomal Cdc20-APC pathway controls dendrite morphogenesis in postmitotic neurons. Cell. 2009;136(2):322-336.

45. Nam HJ, van Deursen JM. Cyclin B2 and p53 control proper timing of centrosome separation. Nat Cell Biol. 2014;16(6):538-549.

46. van den Bos H, et al. Quantification of aneuploidy in mammalian systems. Methods Mol Biol. 2019;1896:159-190.

47. Aziz K, et al. Ccne1 overexpression causes chromosome instability in liver cells and liver tumor development in mice. Gastroenterology. 2019;157(1):210-226.e12.

48. Kanakkanthara A, et al. Cyclin A2 is an RNA binding protein that controls Mre11 mRNA translation. Science. 2016;353(6307):1549-1552.

49. Maskey RS, et al. Spartan deficiency causes genomic instability and progeroid phenotypes. Nat Commun. 2014;5:5744.

50. The Gene Ontology Consortium. Expansion of the Gene Ontology knowledgebase and resources. Nucleic Acids Res. 2017;45(D1):D331-D338.

51. Binns D, Dimmer E, Huntley R, Barrell D, O’Donovan C, Apweiler R. QuickGO: a web-based tool for Gene Ontology searching. Bioinformatics. 2009;25(22):3045-3046. 